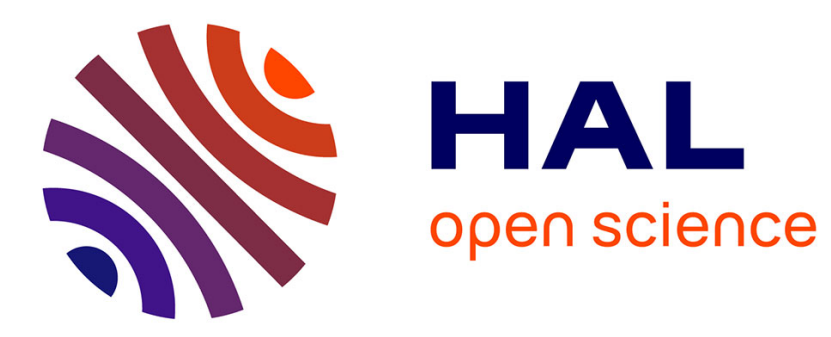

\title{
Glanum et les origines de l'art romano-provençal. Seconde partie: sculpture
}

Gilbert-Charles Picard

\section{To cite this version:}

Gilbert-Charles Picard. Glanum et les origines de l'art romano-provençal. Seconde partie: sculpture. Gallia - Fouilles et monuments archéologiques en France métropolitaine, 1964, 22 (1), pp.1-21. 10.3406/galia.1964.2186 . hal-01934369

\section{HAL Id: hal-01934369 \\ https://hal.science/hal-01934369}

Submitted on 25 Feb 2020

HAL is a multi-disciplinary open access archive for the deposit and dissemination of scientific research documents, whether they are published or not. The documents may come from teaching and research institutions in France or abroad, or from public or private research centers.
L'archive ouverte pluridisciplinaire HAL, est destinée au dépôt et à la diffusion de documents scientifiques de niveau recherche, publiés ou non, émanant des établissements d'enseignement et de recherche français ou étrangers, des laboratoires publics ou privés.

\section{(이) $\$$}

Distributed under a Creative Commons Attribution - NonCommercial - NoDerivatives| 4.0 


\title{
GLANUM ET LES ORIGINES DE L'ART ROMANO-PROVENÇAL \\ SECONDE PARTIE : SCULPTURE
}

\author{
par Gilbert-Charles PICARD
}

Dans la première partie de cette étude je me suis eflorcé d'établir une chronologie des monuments de Glanum, en réunissant toutes les données stratigraphiques, épigraphiques et architecturales dont nous disposons. Cette recherche serait d'ores et déjà à compléter, en tenant compte notamment du récent article de Miss .J. Toynbee, Early Roman Corin$t_{\text {thian }}^{2}$, qui étudie principalement la formation de la corniche corinthienne; on trouverait là évidemment des éléments de comparaison essentiels pour la datation des sanctuaires glaniques; encore faudrait-il que fussent d'abord publiés les membres architecturaux, et en particulier les nombreux fragments de corniche qui ont été retrouvés au sanctuaire de Valeludo et dans les deux temples géminés. Mais il convient, en attendant, de considérer le décor figuré des monuments de Saint-Rémy; à celui des Antiques, justement célèbre depuis longlemps, s'ajoutent maintenanl, comme on l'a dit en commençant, les reliefs triomphaux d'une fontaine et d'un autel situés sur la place triangulaire, un peu au sud du Forum. Cies deux monuments posent de nombreux problèmes qu'il ne sera possible d'examiner sérieusement que lorsque tout le matériel archéologique aura été, là encore, publié ${ }^{3}$.

La fontaine (fig. 1) appartient à une famille de monuments triomphaux à laquelle se rattachent le fornix de Scipion l'Africain, deux ares du ${ }_{1}{ }^{\text {er }}$ s. à Pompéi, l'arc de Caracalla à Volubilis, et le nymphée d'Alexandre Sévère, tous complètés par des bassins ou des vasques 4 . La façade des édicules d'où jaillissait l'eau dans les jardins de Pompéi ressemble à un petit arc, et cette ressemblance est soulignée par le décorateur qui place volontiers dans les écoinçons des Victoires ou des Éros porteurs de vexilla ou de trophées ${ }^{5}$. Inversement, c'est peut être le souvenir de la fonction hydraulique des fornices qui explique la présence à peu près constante dans le décor des ares de divinités fluviales. Cependant les arcsfontaines sont rares à l'époque impériale, avant le III ${ }^{\mathbf{s}}$., et les fontaines triomphales tout

(1) Cf. Gallia, XX1, 1963, p. 111-121.

(2) Journ. Rom. Studies, LII I, 1963, p. 73-84

(3) Notamment les chapiteaux et la corniche de la fontaine signalés dans Gianum II, p. 37-38.

(4) Sur ces monuments, G. Spano, L'Arco trionfale di P. Cornelio Scipione Africano, dans Mem. Acc. Lincei, 1950, 8, 3, p. $173 \mathrm{sqq} . ;$ C. Domergue, L'Arc de Iriomphe de Caracalla à Volubilis, dans Annuaire Ec. Haules Eludes, IV e Seclion, 1963-1964, p. 290-291.

(5) Par exemple la fontaine de la Casa dell'Orso, H. Stern, Etudes archéologiques de Nancy, II, 1959, pl. XXIII, 4. 
à fait exceptionnelles. Aussi la présence de ce monument à Gílanum demeurerait-elle surprenante, si elle ne trouvait son explication dans la religion locale : analogue, dans sa forme et ses dimensions, à des "grottes héracléennes ", comme celle d'Anzio ${ }^{6}$, la fontaine triomphale était sans doute consacrée à Hercule, dieu de la victoire et des eaux salutaires, qui possédait dans le défilé un "fanum " où les vétérans glaniques venaient rendre grâces pour l'heureuse terminaison de leurs campagnes. La fontaine pouvait être alimentée par un canal ayant son origine au nymphée, comme celui qui, selon une heureuse conjecture de M. Rolland, amenait l'eau guérisseuse dans le portique aux incubations, au s.-o. de la place ${ }^{7}$. Les sculptures triomphales seraient en ce cas un ex-voto au dieu. M. Rolland a retrouvé, on le sait, deux

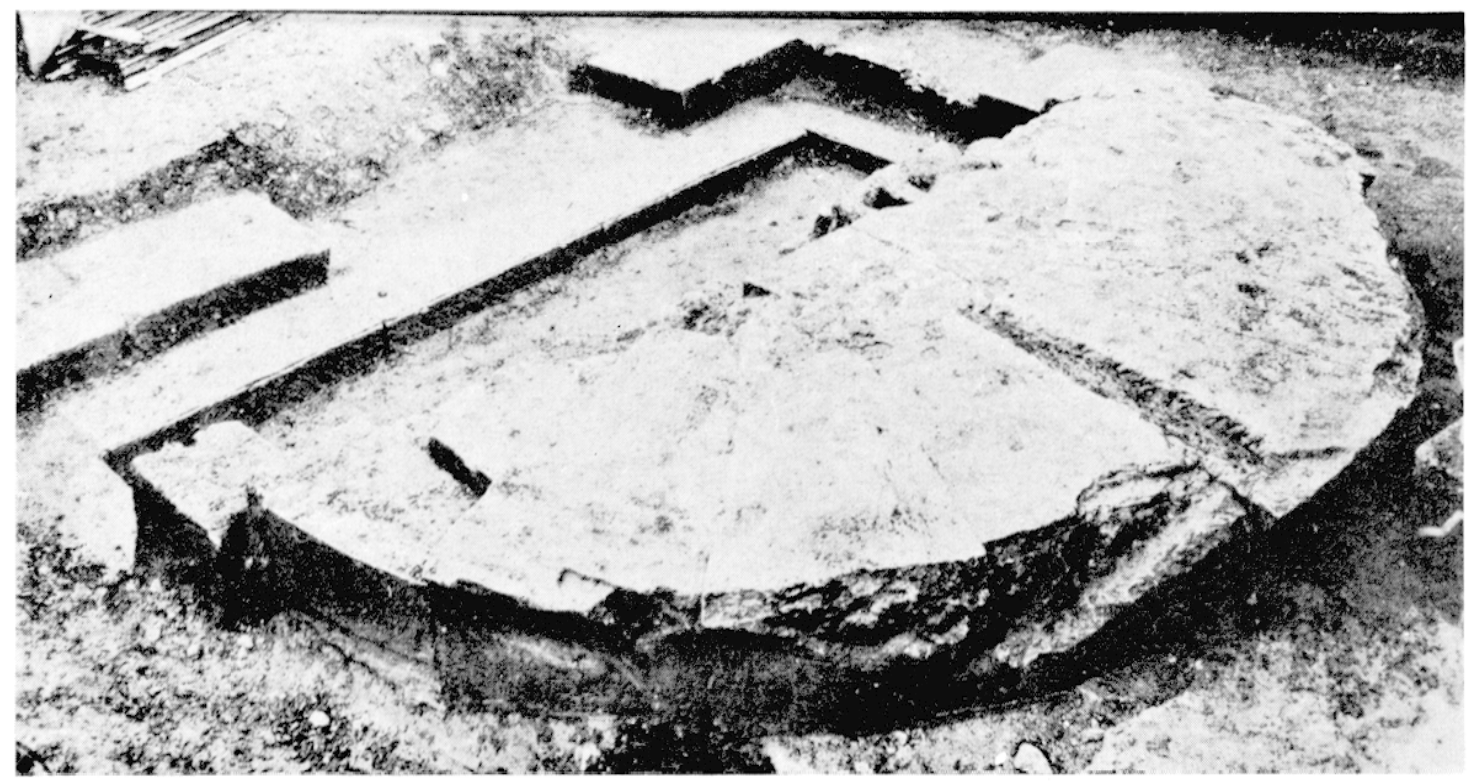

1. - Glanum. Ruines de la fontaine triomphale 'd'après II. Rolland, Glanum II, pl. 9, 2).

statues de captifs agenouillées (fig. 2) et deux cuirasses également traitées en ronde bosse; il a fort justement noté que ces éléments étaient faits pour se correspondre symétriquement et devaient être alignés de part et d'autre d'un motif central unique. Le groupe ainsi constitué avait certainement sa place à l'intérieur de l'exèdre voûtée dont l'actuelle fondation semicirculaire est le vestige. La restitution architecturale de cette partie de la fontaine est malaisément concevable. II. Rolland a songé à un édicule à fronton; mais en ce cas la niche eût été excavée dans un massif de maçonnerie rectangulaire; une demi rotonde voùtée en cul de four, comme dans la maison de M. Lucretius à Pompéi, paraîtrait plus vraisemblable ${ }^{8}$. Pouvons-nous reconstituer l'élément central manquant du groupe ? On attendrait une statue du dédicataire du monument, qui est vraisemblablement Hercule ; il existe préci-

(6) D. Joly, Mel. Ecole fr. de Rome, LXXIV, 1962, 1, p. 123-169.

(7) Glanum II, p. 73-74. Le culte de Silvain parait avoir été localisé près de ce portique, ce qui apporte un argument supplémentaire en faveur de notre hypothèse, en raison de la parenté des deux dieux dont les monuments étaient aussi rapprochés.

(8) Je me suis servi pour cette recherche de l'excellent mémoire de M $^{11 e}$ Joly, consacré aux fontaines de Pompeï, et de son article cité ci-dessus n. 6 . 
sément un type d'IIercules Victor, assis entre deux cuirasses, qui apparaît notamment sur un des médaillons hadrianiens de l'are de Constantin, et diverses monnaies du II $^{\mathrm{e}}$ s. J'avais d'abord songè à en proposer la restitution au centre de la fontaine de Glanum, mais diverses difficultés m'ont fait renoncer à cette hypothèse. Tout d'abord, IIme Squarciapino, qui a étudié ces figurations d'Hercule ${ }^{9}$, note qu'aucune n'est antérieure à Hadrien et attribue à cet empereur la dédicace de la statue de culte qu'elles reproduisent. D'autre part, aucune des répliques du groupe ne comporte de captifs agenouillés. Ceux-ci sont presque toujours associés à un trophée; le thème. qui existait sans doute déjà dans l'art hellénistique - à Pergame notamment - apparaît dès l'an — 100 dans la numismatique romaine avec les quinarii de C. Fundanius et T. Cloulius qui célèbrent les victoires sur les Cimbres ${ }^{10}$, et on les retrouve ensuite sur les revers de l. Memmius vers - $56^{11}$, puis quelques années plus tard sur de célèbres monnaies césariennes ${ }^{12}$. L'élément manquant du groupe de Glanum pourrait donc être un trophée. Ln fragment de cuirasse retrouvé par II. Rolland en serait l'unique vestige. Les deux cuirasses conservées ne sont pas elles-mêmes des trophées; elles sont posées à terre, soutenues par une sorte de porte-manteau qui servait à les conserver lorsque leur propriétaire n'en faisait pas usage (fig. 3 et 4). On voit des cuirasses ainsi présentées au pied de statues du I ${ }^{\text {er }}$ s. av. . J.-li. ; l'exemple le plus connu est l'effigie d'époque syllanienne de Tivoli, conservée au Musée des Thermes, qui représente peut être A. Postumius Albinus ${ }^{13}$. Il est rare, mais non sans exemple, qu'une cuirasse soit ainsi dressée au pied d'un trophée; c'est le cas sur l'une des fresques de l'Armentarium de Pompéi, qui reproduisaient à mon avis les trophées de César. La forme des cuirasses apporte un important élément de datation; elles se rattachent à un type hellénistique dont les exemples les plus connus appartiennent au Mithridate et au Bilienus de Délos, au Mars - ou général romain de la base dite d'Ahenobarbus, au Postumius Albinus de Tivoli( ?), ou sont reproduits par des monuments funéraires du Dodécanèse, les frises de Pergame et la frise syllanienne de la Via della Cionsolazione au Capitole". Toutes ces armures "en baril" sont formées par un corselet métallique arrêté au niveau du diaphragme, dont le plastron et le dosseret sont réunis par des épaulières descendant au moins jusqu'aux seins; des lambrequins de cuir protègent les cuisses. Les cuirasses de Glanum présentent un certain nombre de particularités : 10 l'encolure est formée par un gorgerin saillant en bourelet; on retrouve cet élément ${ }^{15}$ sur deux cuirasses de la frise de Pergame et sur celle d'un trophée en ronde bosse découvert sur le même site, toutes trois du type "long et musclé ", ainsi que sur le camée portrait de Ptolémée Philadelphe ${ }^{16}$. Les cuirasses à gorgerin, longues ou courtes, apparaissent également a) sur une monnaie de lésar et une monnaie actienne d'Octave ${ }^{17}$;

(9) Ln lipos slaluario di IIercules Invichus, dans Bull. Comm. LXXIII, 1949-50, p. 205-214.

(10) E. A. Sydenham, Rom. Rep. Coinage, p. 81, nos 584 et 586.

11) Ibid., p. 153 et pl. 25, no $^{\circ} 920$.

(12) Ibid., p. 167-169. ( $\mathrm{Xi}$ la date, ni le lieu d'émission de ces monnaies ne nous semblent encore établis de manic̀re absolument satisfaisante.)

(13) B. Felleti Maj, I Rilralli del Museo Naz. Romano, p. 33 sqq., no 45.

(14) Trophées Romains, p. 220 sqq et pl. VII, 2.

(15) En dernier lieu, C. C. Vermeulle, Hellenislic and Roman Cuirassed Slatues, Berytus, XIII, 1, 1959, p. 13 sqq.

(16) P. Couissin, Rev. Arch., 1923, 1I, p. 81-82.

(17) Monnaie de César, E. A. Sydenham, op. l., p. 169 no 1016 ; Trophées Rom., p. 220 et pl. VIII. Monnaie d'Octave, Mattingly Sydenham, RIC, I, p. 63, n³3. Cf. Trophees Romains, p. 256-257 et pl. VIII. 


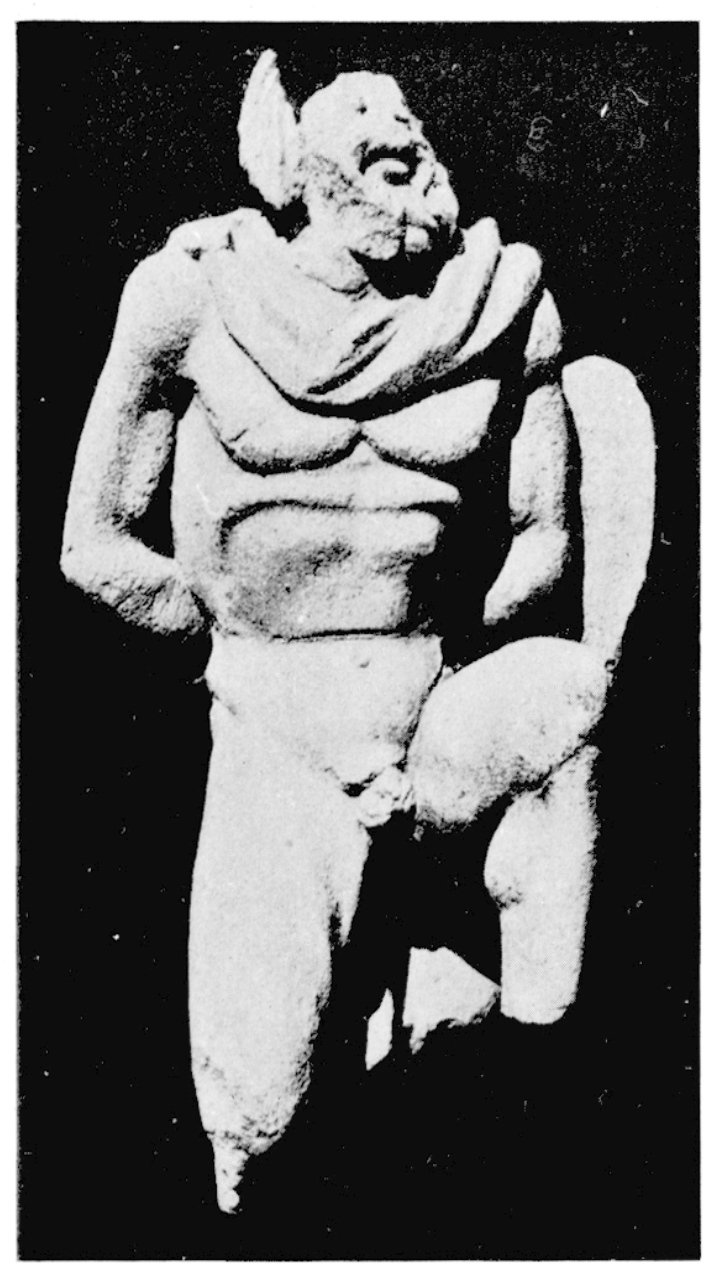

2. -... Glanum. Fontaine triomphale. Statue de captif (d'apries II. Rolland, Filanum II, pl. 10, 1).

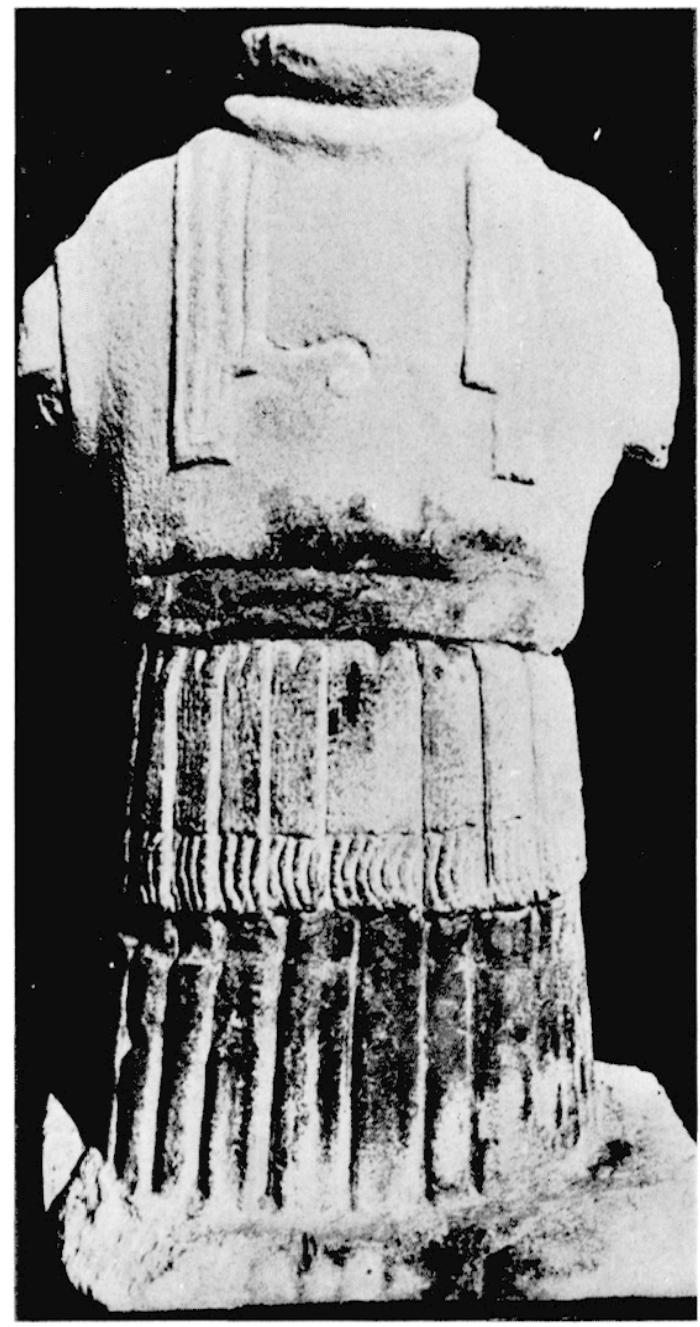

3. . Gianum. Cuirasse de la fontaine triomphale d'apres H. Rolland, Gilanum II, pl. 10, 2\%.

b) sur l'are de Pola et le relief de Ielminium ${ }^{18}$. qui commémorent probablement la campagne illyrienne de -35 - $-33 ; c$ ) en Gaule sur le cippe de Tauroentum et les reliefs de Narbonne. 20 Les épaulières très longues, sont échancrées au bas vers l'intérieur, au lieu de l'être vers l'extérieur selon la coutume; cette anomalie, très rare, ne se retrouve à notre ronnaissance que sur un petit trophée portatif en bronze (fig. 6 ), découvert précisément à (ilanum par .I. Rolland ${ }^{19}$, et qui devait appartenir à une statue de Victoire, de Mars de Minerve ou d'Hercule, puisque ces divinités sont seules porteuses de trophées ${ }^{20}$. Ce trophée se compose d'un casque à cimier phrygien, analogue à celui que portent plusieurs personnages du mausolée, et d'une cotte d'armes en cuir; j'avais d'abord cru qu'il datait du

(18) Trophees Rom., p. 232, pl. XII, 2. Les rinceaux flanquant le cartouche de l'inscription sont analogues a ceux du temple de Divus Iulius.

(19) Cf. Trophées Rom., p. 199, n. 3. C'est par erreur que j'ai parlé là d'un casque du type de Haguenau. différent.

(20) Ibid., p. 126-128. Il ne peut s'agir d'une statue de Romulus, le trophée porté par le roi étant d'un type 


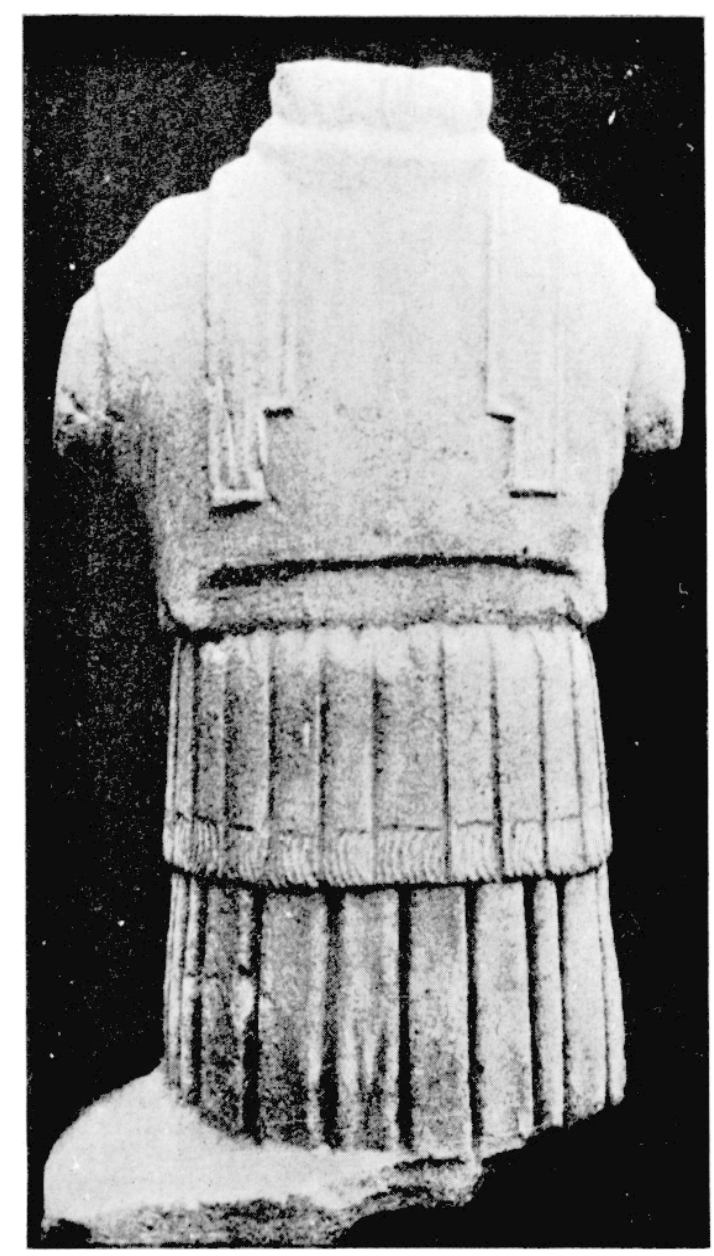

4. - Glanum. Cuirasse de la fontaine triomphale (d'après II. Rolland, Glanum II, pl. 10, 3).

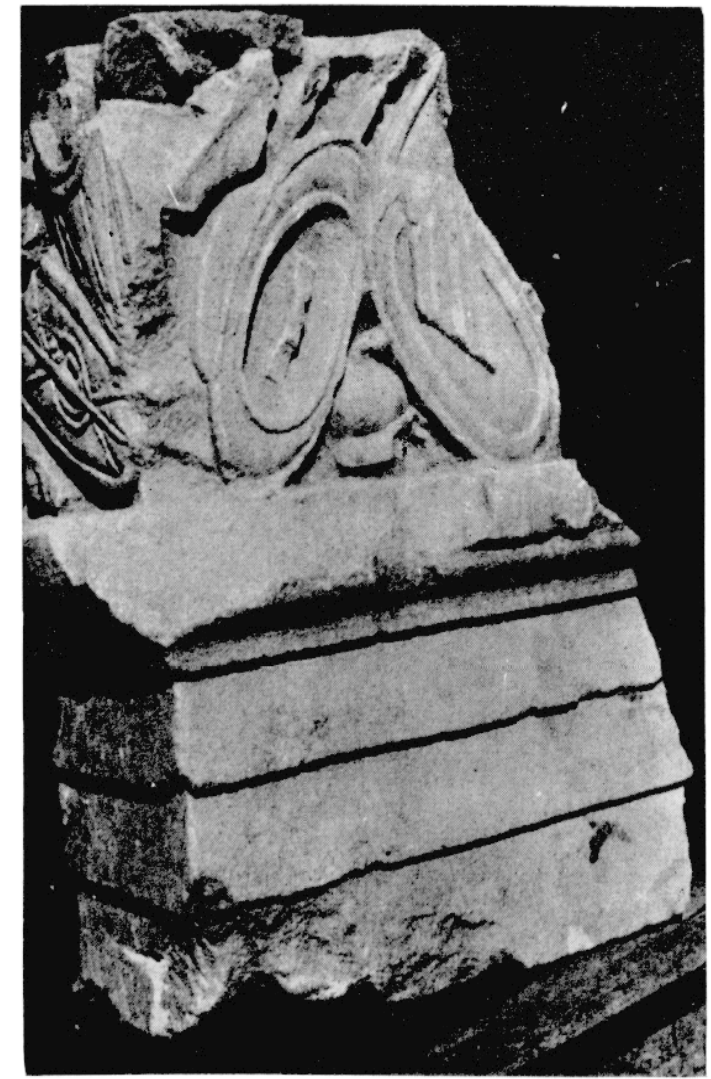

5. - Glanum. Entablement de la fontaine triomphale. Frise d'armes (d'apres H. Rolland, Glanum II, pl. 10, 1).

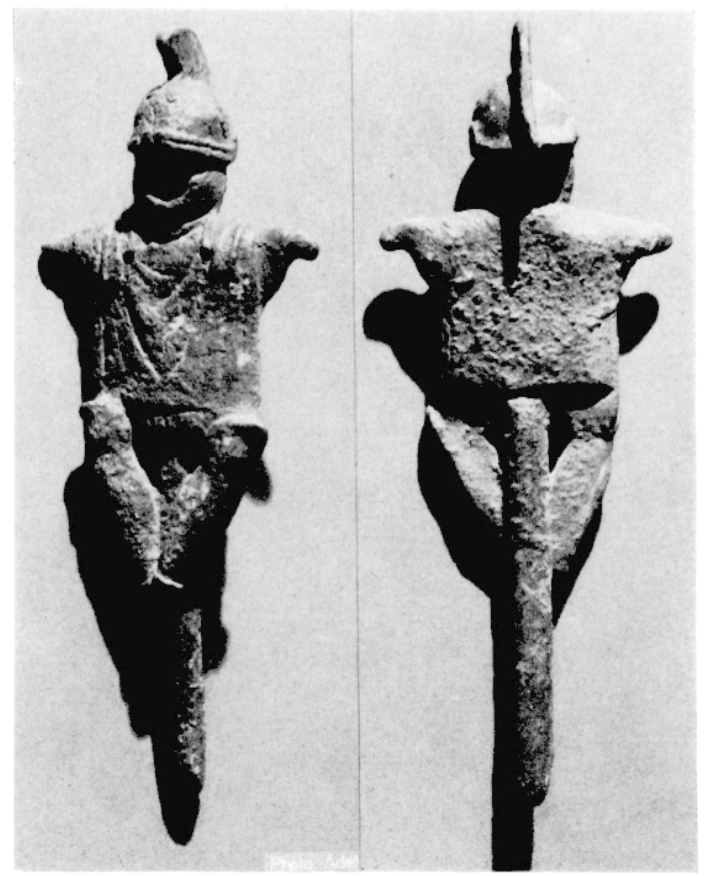

6. - Glanum. Trophée portatif en bronze. (Photo H. Rolland). 
$+\mathrm{II}^{\mathrm{e}}$ s., cette sorte d'armure apparaissant sur les monuments de cette époque, mais la concordance entre la forme des épaulières et celle des cuirasses de la fontaine est un indice suffisant pour établir que cet objet est contemporain de l'ensemble des grands monuments de Glanum. A. Hekler21 a montré que la diminution progressive de la taille des épaulières fournissait un moyen de dater les cuirasses. Le seul monument postérieur à Actium qui présente des épaulières aussi grandes que les cuirasses de Glanum est la statue de Vachères que le type du visage permet de dater au plus tôt de -20); mais l'armure est ici une cotte de mailles. $3^{0}$ Le plastron de l'une des cuirasses est dépourvu de tout décor; l'autre porte une seule volute horizontale incisée sur la moitié droite ; je ne connais pas d'autre exemple de cet ornement qui semble rajouté après coup ; mais on peut se demander si son auteur n'a pas voulu imiter maladroitement, soit le rebord d'une égide analogue à celle que porte le général d'époque triumvirale de la Glyptothèque de $\mathrm{Munich}^{22}$, soit un serpent placé sous un gorgoneion ; en ce cas le décor n'aurait pas été achevé ${ }^{23} ; 4^{0}$ les deux cuirasses présentent une sorte de gouttière au bas du corselet ; là encore je ne connais pas d'élément de comparaison. Ciette goultière servait à maintenir en place le ceinturon ; mais celui-ci est généralement placé plus haut; ;0 il n'y a qu'une série de lambrequins, sous laquelle apparait le bas d'une tunique plissée ; le sculpteur a nettement marqué la différence entre les coupures qui séparent les languettes frangées et les plis de l'étoffe ou du cuir qui constitue le vêtement. En conclusion, les cuirasses appartiennent à un type hellénistique qui disparaît peu après Actium. Leurs anomalies peuvent s'expliquer soit par la maladresse du sculpteur soit plutôt par l'origine des armes elles-mêmes, qui pourraient ètre des copies barbares d'un modèle grec.

Venons-en maintenant à la frise d'armes qui faisait partie de l'entablement de l'exèdre ${ }^{24}$ (fig. 5). On aperçoit sur la face antérieure deux boucliers ovales dont l'un montre sa face interne ; un casque est posé entre eux ; trois lances ou javclots dépassent en arric̀re ; une cnémide est présentée de face ${ }^{25}$; sur le retour sont sculptés un bouclier ovale, un autre en forme d'ècu cintré, et un troisième objet indistinct, peut être un casque.

L'élément de frise, provenant sans doute de l'autel monumental, est fort analogue ${ }^{26}$ (fig. 7). Un captif agenouillé dans la même posture que les prisonniers en ronde bosse de la fontaine occupe l'angle. Dans l'amoncellement de dépouilles qui meuble le reste du champ, se discernent de gauche à droite et de bas en haut : la moitié d'une pelte, un bouclier circulaire bombé, un second bouclier analogue, un casque, le pommeau d'un glaive, un bouclier hexagonal qui montre sa face interne.

Cies deux frises sont évidemment inspirées de la balustrade d'Athéna Polias à Pergame. Il existe un autre type de frise d'armes, d'inspiration néoattique, avec dépouilles ${ }^{27}$; un seul monument provençal, de facture d'ailleurs très médiocre, s'en inspire : c'est le pseudo

(21) JOAI, XIX-XX, 1919, p. 190 sqq.

(22) B. Schweitzer, Bildniskunsl, p. 65 ; C. C. Vermeulle, „p. l., p. 34, pl. III, fig. 10.

(23) Comparer les ornements en spirale, dérivant d'égides déçénérées, qui ornent les pectoraux des guerriers d'Entremont.

(24) Glanum II, p. 38, pl. 10, 1.

(25) Et non un frontal de cheval comme le croit $H$. Rolland.

(26) Gilanum II, p. 43, pl. 11, 2.

(27) Cf. les frises de la Via della Consolazione et du Kbor Klib (MEFR, LXXI, 1959, p. 263 sqq). 
trophée de Biot ${ }^{28}$. Les amoncellements de type pergaménien sont au contraire fréquents dans l'art romano provençal ; en dehors des panneaux de l'arc d'Orange, dont la composition est originale ${ }^{29}$, il existe des débris de ce genre de reliefs à Marseille (" cippe de Tauroentum »), Arles, Avignon, et Vienne, pour nous en tenir à ceux qui peurent être datés avec quelque vraisemblance du début de l'Empire ${ }^{30}$. Nous ne connaissons pas au contraire de frises d'armes amoncelées à Rome ou en Italie péninsulaire qui remontent plus haut que le milieu du ${ }^{\text {er }}$ s. Mais la frise de l'arc de Pola et le relief déjà cité de Delminium sont proches des monuments provençaux.

A quel peuple appartiennent les dépouilles et les captifs de Glanum ? Les vaincus nus, agenouillés au pied d'un trophée qu'avaient sans doute sculplés les artistes pergaméniens, étaient des Galates. On retrouve des Nordiques ou des Celtes sur les monnaies de Cloulius, Fundanius et César, ainsi que sur le relief d'Isernia ${ }^{31}$, et à Saint Bertrand-de-liomminges. Mais le denier de Memmius représente un Asiatique dans la même posture. A I Delminium il s'agit d'Illyriens. Aucune des armes de la fontaine n'est spécifiquement celtique; les Gaulois utilisaient le bouclier ovale, comme d'ailleurs les légionnaires jusqu'à l'époque d'Auguste; le thyreos sur lequel s'appuie le guerrier agenouillé pourrait être gaulois. Mais ceux de la frise paraissent plus petits, plus fortement bombés, avec un rebord plus large et plus saillant; ils ressemblent aux thyreoi des frises de Pergame, et d'autre part à ceux que portent les soldats embarqués sur la birème de Préneste; nous croirions volontiers qu'ils sont faits de métal. L'autre bouclier de la frise de la fontaine a la forme d'un écu triangulaire et cintré ; ce type d'arme était utilisé par les populations du nord des Balkans ; à l'époque d'Auguste nous le trouvons sur le relief de Delminium, où il voisine avec des thyreoi ovales analogues à ceux de Glanum et des rondaches, et au bas du trophée de Tibère sur la Gemma Augustea. Le trophée du relief de Delminium comporte des cnémides, comme celle qui se détache près de l'angle de notre frise (fig. 8).

Le casque sculpté sur la frise de Glanum est très voisin de celui que portent les légionnaires du "relief d'Ahenobarbus "; ce casque à bombe conique, visière débordante et larges garde-joues, coifle également les trophées de la via della Consolazione; d'origine italique, il a été emprunté par des peuples voisins, en particulier par les Gaulois ${ }^{32}$. On ne trouve sur la frise de la fontaine ni le carnyx qui ne manque jamais dans les monuments des défaites gauloises, ni l'épée longue de la Tène II, ni le bouclier plat polygonal, ni les casques ornés de cornes et de rouelles. Il est donc presque certain que les dépouilles n'ont pas élé enlevées à un peuple celte.

La frise de l'autel comporte au contraire deux armes indubitablement celtiques : un casque du type Alise-Agen ${ }^{33}$, et un bouclier polygonal. Elles sont associées à des rondaches

(28) Trophées Romains, p. 150 sqqq.

(29) G. Ch. Picard et J. J. Hatt, L'Arc d'Orange, p. 77 sqq.

(30) Cf. Trophees Romains, p. 195-196. Pour Vienne, en outre I. Will, La sculplure romaine au Musee de Vienne, p. 61-63, no 120. J'avais daté le cippe dit de Tauroentum d'une époque plus tardive; mais le type des armes (cuirasse à gorgerin) est bien celui des monuments augustéens. I.a frise de la fontaine de Gilanum est d'un travail beaucoup plus soigné que la plupart des autres reliefs d'armes provençaux; elle a été sculptée au ciseau, sans emploi du trépan.

(31) H. Fuhrmann, MDAI, II, 1949, p. 45-65, pl. 11-13.

(32) P. Couissin, Armes Romaines, p. 327.

(33) J. Déchelette, Manuel d'Archéologie, II, p. $1165-1167$. 
bombées rappelant l'aspis macédonienne ${ }^{34}$, et à une pelte amazonienne. Le pommeau de poignard ou de glaive est probablement romain ${ }^{35}$.

Il semble donc que les deux monuments triomphaux célèbrent des victoires différentes, l'autel seul étant en rapport avec une défaite gauloise. Cependant avant de risquer une hypothèse sur la nationalité des vaincus de la fontaine il convient de comparer leur armement avec celui des guerriers du mausolée.

Cie monument - dont nous appelerons par la suite le sculpteur Glanicus - présente comme on sait trois scènes de batailles : au s.-e. un combat de cavaliers et de fantassins (fig. 9-10); au n.-e. trois duels de cavaliers (fig. 11) ; au n.-0. un combat de fantassins autour d'un cadavre (fig. 12). Tous ces tableaux dérivent de poncifs : dans le premier le groupe central (guerrier tirant un cavalier ou une cavalière à bas de son cheval) se rencontre fréquemment dans les amazonomachies. Au n.-o. le sujet principal est inspiré par la dispute du cadavre de Patrocle. Le prototype du tableau n.-e. n'avait pas été retrouvé jusqu'ici ; or la figure centrale (cavalier vu de dos, sabrant un adversaire qui l'assaille à droite) se trouve sur un denier frappé par le monétaire C. Servilius, vers 120 av. J.-C.. ${ }^{36}$ (fig. 13). Ciette monnaic évoque très probablement l'un des duels de Servilius Pulex Geminus, fameux champion romain de la seconde guerre punique ${ }^{37}$. Pulex de profil attaque par le flanc droit un cavalier vu de dos, fort analogue, à quelques détails près sur lesquels nous reviendrons, à celui de Saint-Rémy ; quoique les deux figures soient à peu près identiques, la composition des groupes apparaît donc fort différente puisque les deux cavaliers de Glanum, tournés l'un vers l'arrière et l'autre vers l'avant, sont étroitement imbriqués l'un dans l'autre en une unité indissoluble. On pourrait croire que Glanicus et le graveur de la médaille n'ont d'autre lien que d'avoir emprunté indépendamment un même poncif à un cahier de modèles. Il faut cependant dépasser cette conclusion négative; E. Garger ${ }^{38}$ nous y aide en observant que les couples de duellistes étroitement embrassés de Saint-Rémy dérivent de ceux des urnes étrusques; ce procédé de composition appartient donc à Glanicus, formé par une tradition italique. Dans le cas présent, le cavalier de droite, retourné comme il est à gauche sur la croupe de son cheval, est manifestement hors d'état de férir son adversaire, et la lance qu'il brandit ne peut atteindre que le vide. Le relief de Saint-Rémy dérive done bien de la monnaie de Servilius ou plutôt de la peinture qu'elle résume, et que Glanicus a redessinée pour des raisons purement formelles. B. Andreae a d'ailleurs démontré que le cavalier frappant de sa lance, en se détournant, un adversaire assis tombé sous son cheval (dernière figure à droite du relief n.-e.) reproduit un poncif du temps d'Alexandre le Grand ${ }^{39}$. Or, c'est aussi à cette époque que doit remonter le prototype qui servit de modèle au graveur

(34) P. Couissin, R. A., 1923, 2, p. 65-68.

(35) Ibid., p. 51, fig. 8 .

(36) A. Sydenham, Roman Republican Coinage, p. 57, n 483. Grueber, I, 1166, datait cette monnaie de -94 . Cf. G. Becatti, Arte Romana, fig. 10.

(37) Cr. Munzer, R.E., s. v. Servilius, n78. Mattingly, cité par Sydenham, croit que la monnaie représente le magister equilum Servilius A hala tuant Spurius Maelius; mais l'armement du cavalier vaincu est celtique (cf. infra).

(38) Röm. Mill., LII, 1937, p. 1-44.

(39) B. Andreae, Molivgeschichlliche Unlersuchungen zu den Römischen Schlachlsarkophagen, p. 20-28. Le type apparaît sous sa forme la plus simple dès la fin du jer siècle. Les sarcophages du groupe Ammendola en présentent la forme la plus évoluée créée par l'art pergaménien. La figure de Saint-Rémy se situe à mi-chemin et s'apparente à la statuette dite d'Alexandre à Naples. 


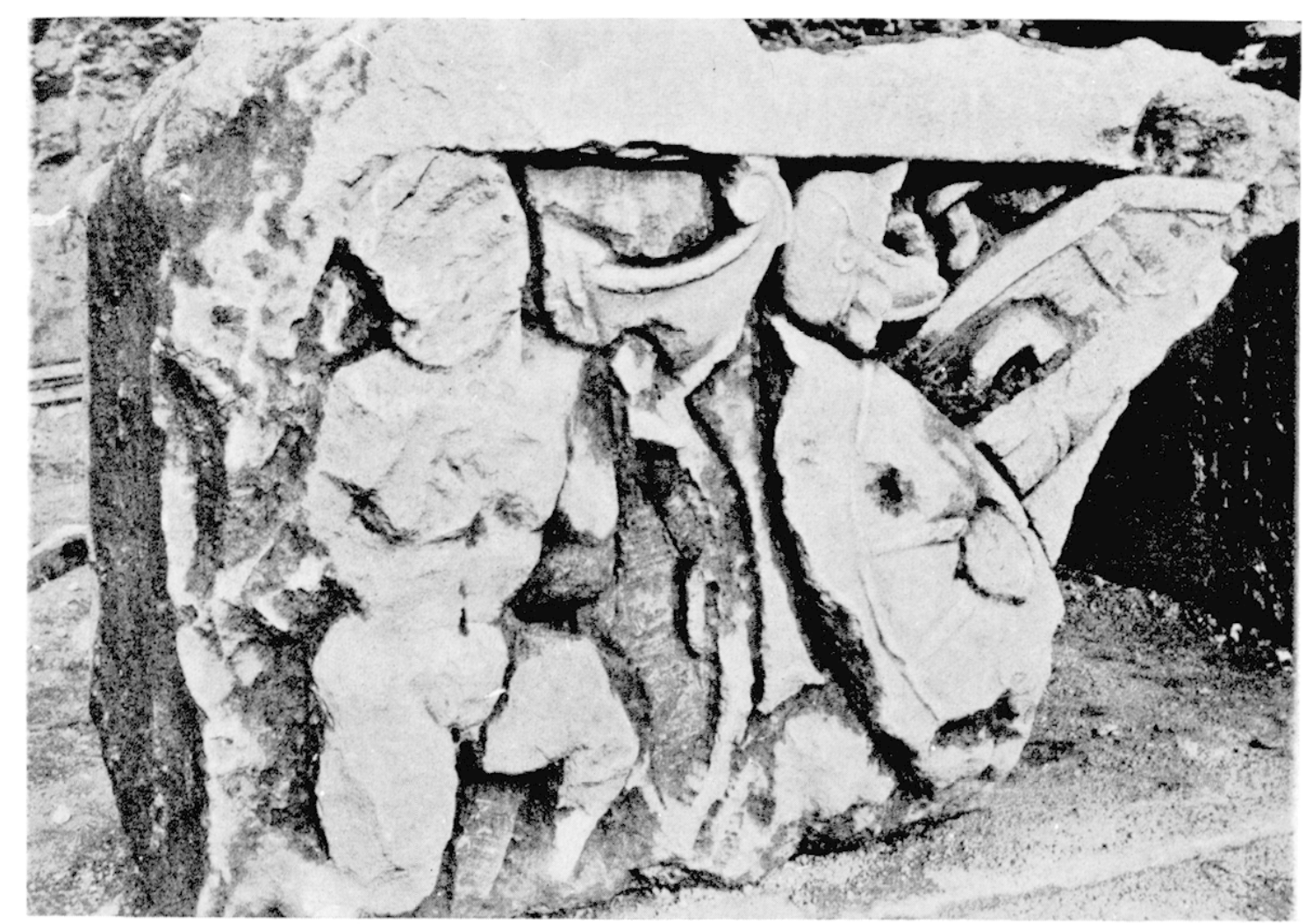

7. -- Glanum. Frise de l'autel triomphal d'apres II. Rolland, Gilanum II, pl. 11, 2;.

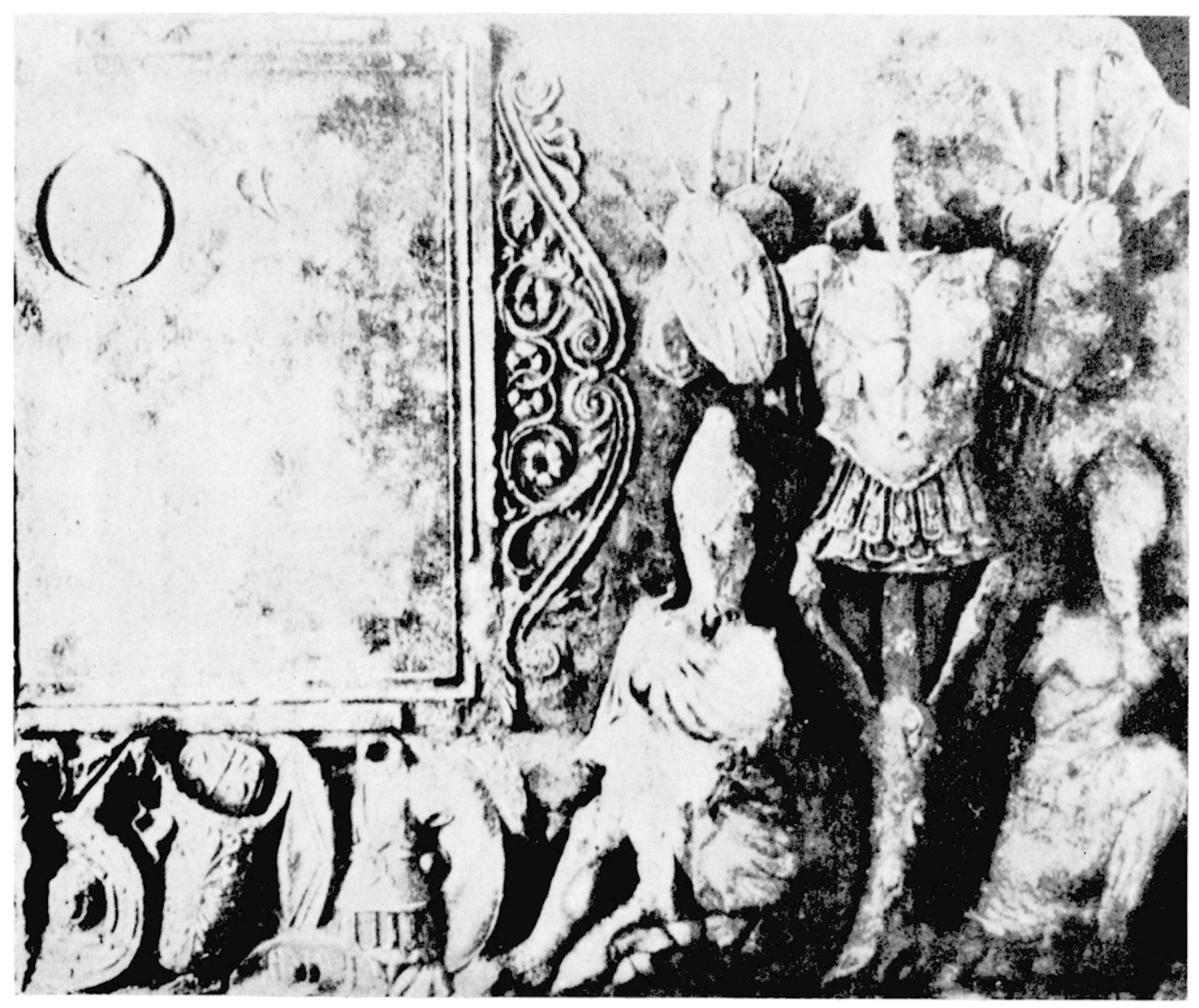

8. - Relief de Delminium. 


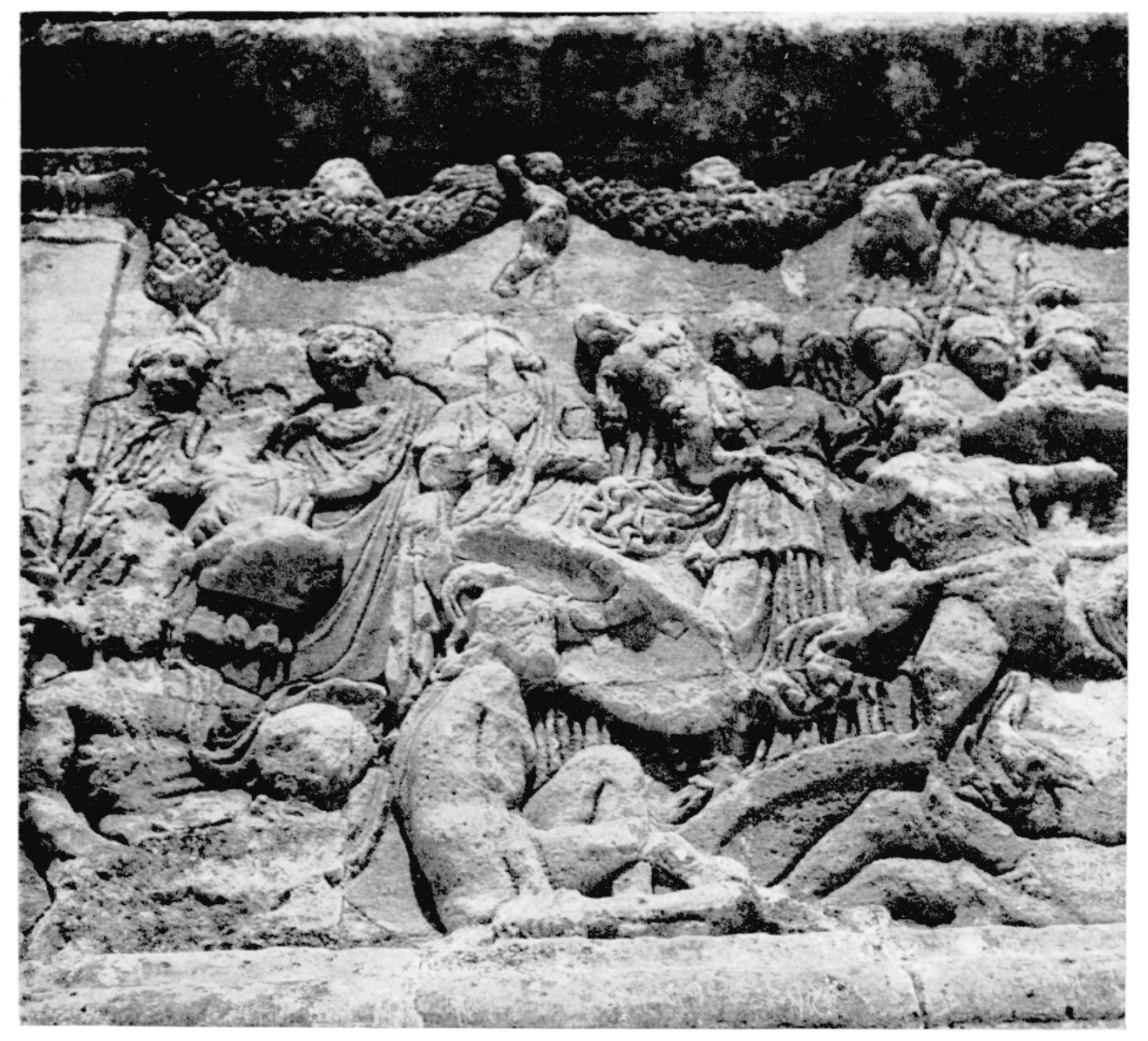

9. - Glanum. Mausolée des Julii. Détail de la face s.-e. (partic gauche). (Pholo Roger Viollet).

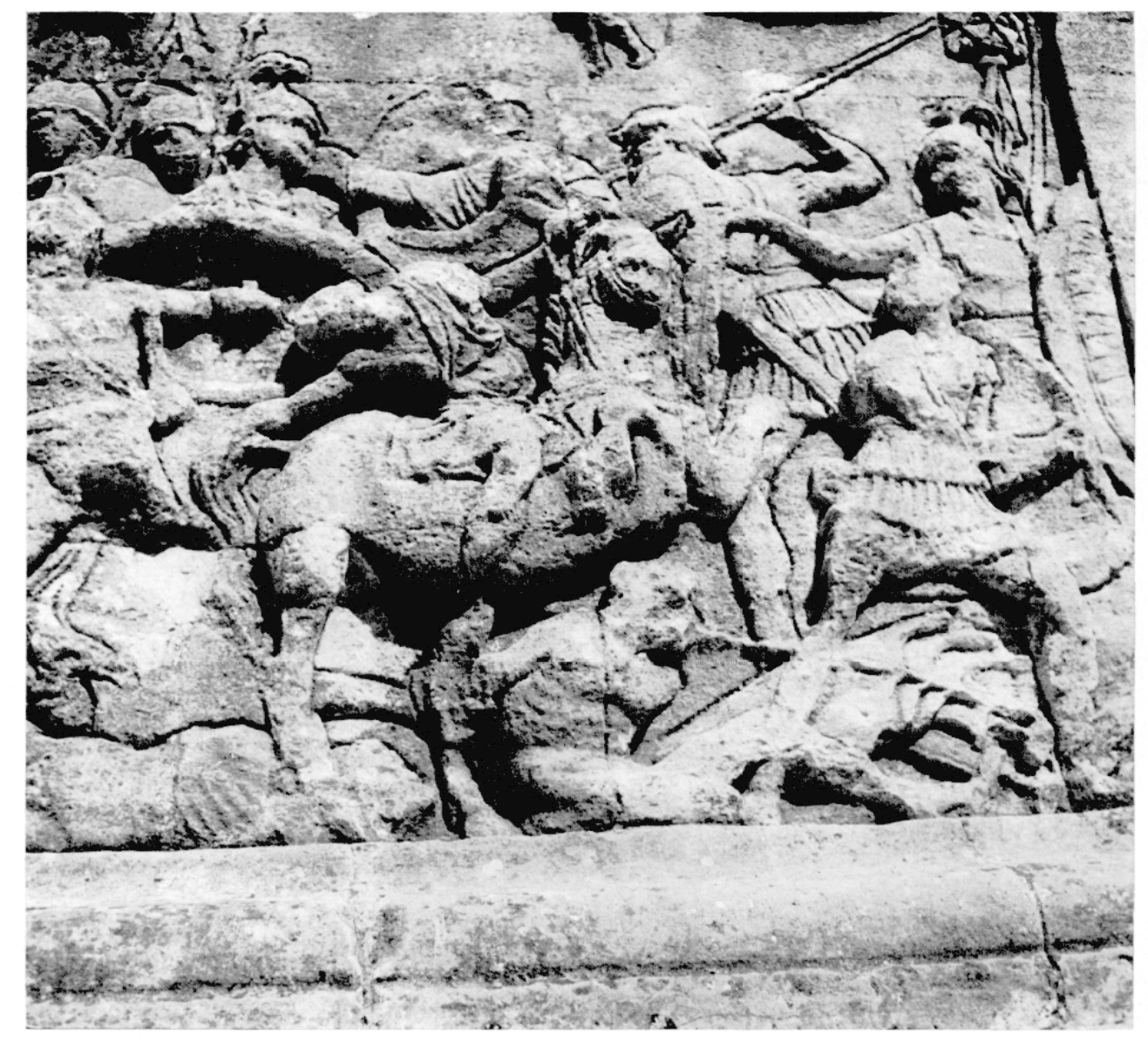

10. - Glanum. Mausolée des Julii. Détail de la face n.-e. (partie centrale). (Photo Roger-Viollet). 


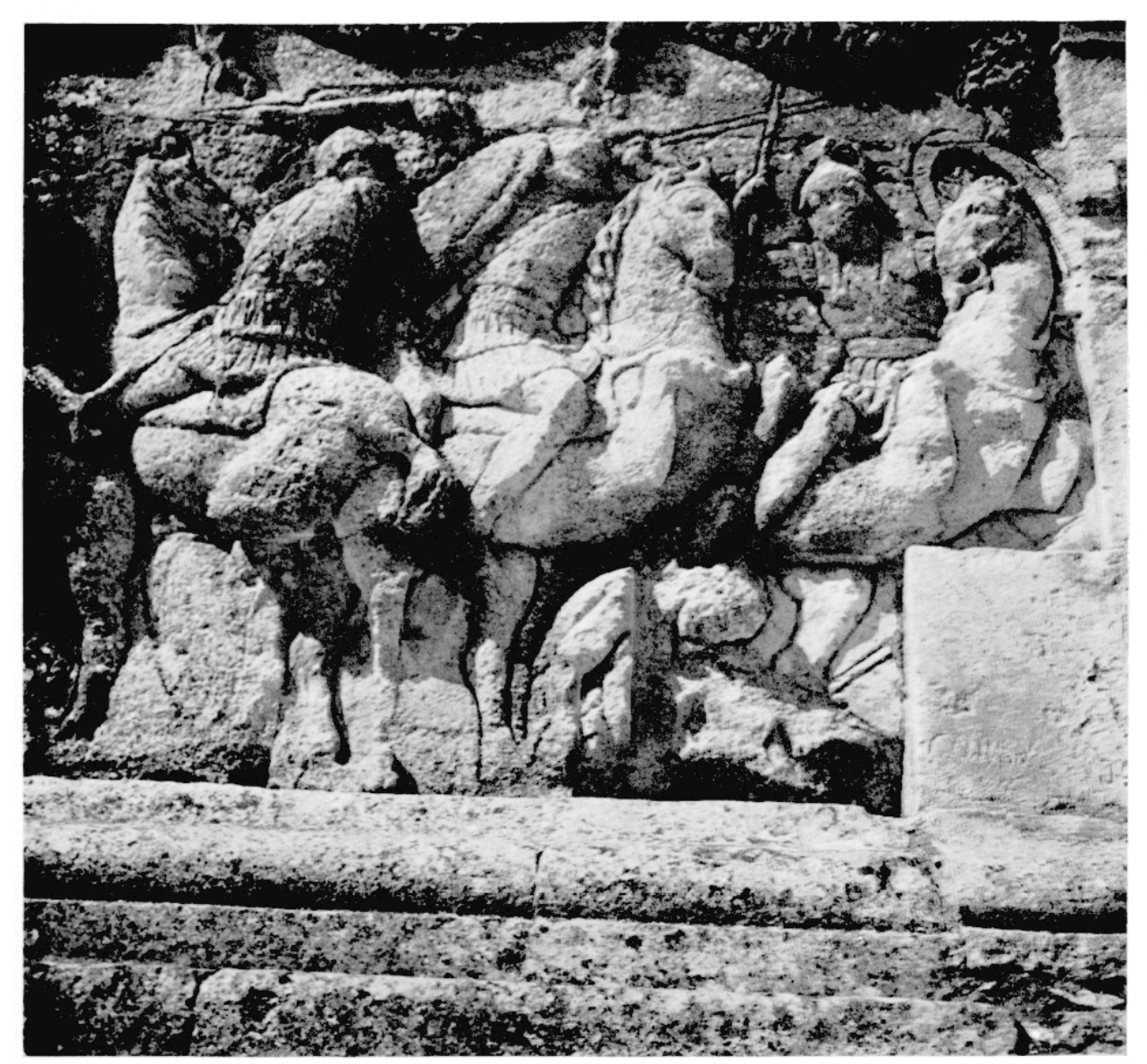

11. - Glanum. Mausolée des Julii. Détail de la face n.-e. (Photo Roger-I'iollet).

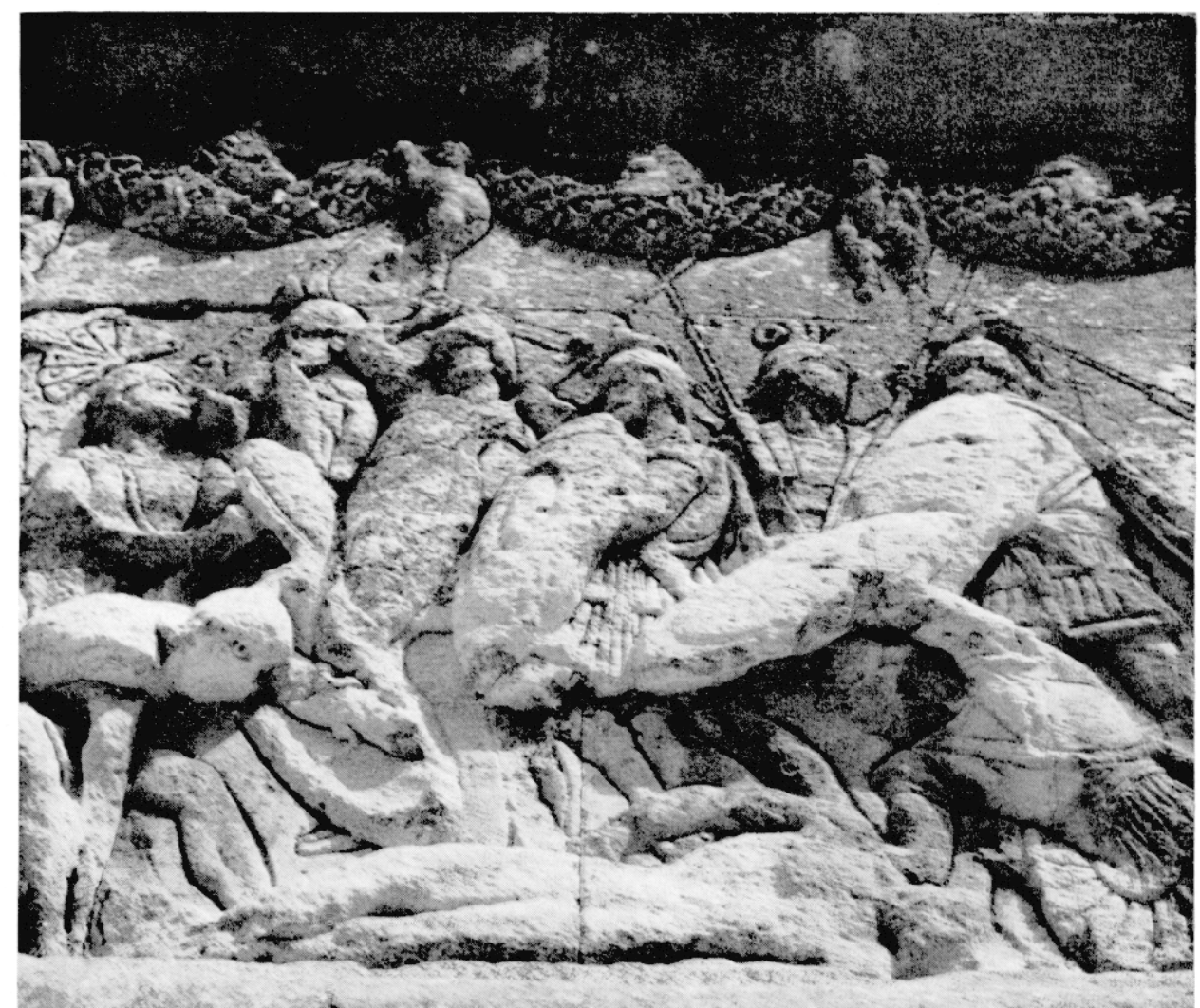

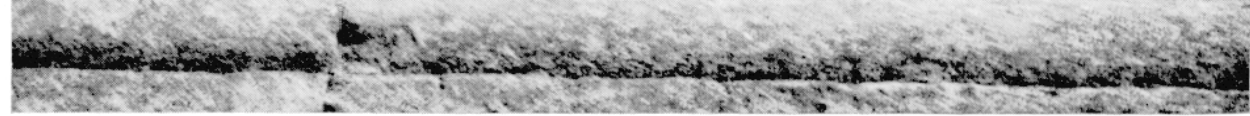
12. - Glanum. Mausolé des .Julii. Détail de la face n.-0. (Phollo Rerger-l'iollel) 
de Servilius : son groupe décalque en effet exactement, en le retournant, l'Alexandre transperçant un Perse de la Casa del Fauno à Pompéi. Nous pouvons donc dès maintenant formuler une première conclusion définitive sur la genèse du relief de Glanum : il ne dérive pas immédiatement d'un modèle grec mais s'inspire d'abord d'une œuvre de la peinture de bataille romaine de la fin du $\mathrm{II}^{\mathrm{e}} \mathrm{s}$., par cet intermédiaire d'un tableau peint aux environs et de -300 .

Mérimée dès $1835^{40}$ avait reconnu dans le bas-relief N.-W. l'illustration du chant XVII de l'Iliade (dispute du cadavre de Patrocle). L'identification, incontestable, a été depuis acceptée par tout le monde; cependant la recherche précise de sources plastiques s'avère difficile; l'épisode n'a pas été retenu par les sculpteurs des tables iliaques. On ne voit pas d'autre part de rapport formel entre le relief de Glanum et le célèbre groupe de la Loggria dei Lanzi ou sa réplique romaine, dite Pasquino, qui représentent Ménélas soutenant Patrocle sur son genou; la liaison existe cependant mais on ne peut la reconnaitre que grâce à l'une des oenochoés de Berthouville ${ }^{41}$. Le toreute a représenté la mort d'Achille, mais en suivant de très près le texte homérique : Ajax soutient Achille défaillant et le couvre de son bouclier. (Blanicus a dédoublé Achille en un cadavre étendu de tout son long et un guerrier agenouillé qui se protège lui-même avec son aspis. Il a conservé Ajax. Vénélas qui accourait par derrière a son secours, s'est lui aussi dédoublé en deux combattants. La Xiké tropeophore s'est muée en un guerrier coiffé d'un casque à cornes. Le Gree qui apparaissait au second plan derrière le bouclier d'Ajax a été triplé ; enfin les trois 'Troyens attaquant par la gauche ont été maintenus, au prix d'un léger décalage; quant au guerrier penché (Hippothoos dans l'Iliade) qui tire par les pieds le cadlavre, il n'existe pas sur le vase de Berthouville, mais on le retrouve sur des camées ${ }^{42}$. Nous décelons donc à nouveau un archétype hellénistique, une peinture sans doute, comme la plupart des modèles des torentes. Mais la transmission s'est, faite là encore par l'intermédiaire italique : nous le reconnaissons à l'orientation des personnages du relief, tournés pour la plupart vers le spectateur sans souci de la clarté de l'action. Le premier "Achille agenouillé " et l' "Ajax " adoptent ainsi une posture que les sculptures d'urnes étrusques prêtaient le plus volontiers aux héros grees: agenouillés de face, le bouclier levé au-dessus de la tête ${ }^{43}$. Ln autre personnage à l'extrémité druite est lui aussi à genoux, mais tourne le dos au spectateur ; parfaitement inutile, il n'a d'autre raison d'être que de former un couple antithétique avec l'“Ajax » exactement comme le cavalier qui a pris la place de Servilius Pulex balance son partenaire. Au lieu de se grouper en deux masses adverses ou de s'opposer en une série de duels, les combattants, à quelque parti qu'ils appartiennent, forment un seul front continu, sinusoïde, dont le mouvement est d'abord a gauche, dirigé d'arriere en avant, puis qui recule en s'arrondissant autour du cadavre, et revient à droite vers le premier plan.

Au S.-E. Glanicus a juxtaposé deux scènes qui semblent sans rapport entre elles; une bataille occupe plus des trois quarts du champ. Elle s'ordonne autour d'un guerrier qui

(40) P. Mérimée, Noles d'un voyjage dans le . Nidi de la France, 18.35.

(41) E. Babelon, Le Trésor de Berthouville, pl. 17. K. L.ehmann, A.JA, Xl.II, 1938, p. 82 sqq.

(42) A. Furtwängler, Ant. Gemmen, pl. 23, 4.

(43) (:f. par ex. les urnes, Brunn et Korte, Rilievi delle Lrne Eitrusche, II, 5, 1. S. Reinach, R. IR., III, 14, 3; 446,2 etc... 


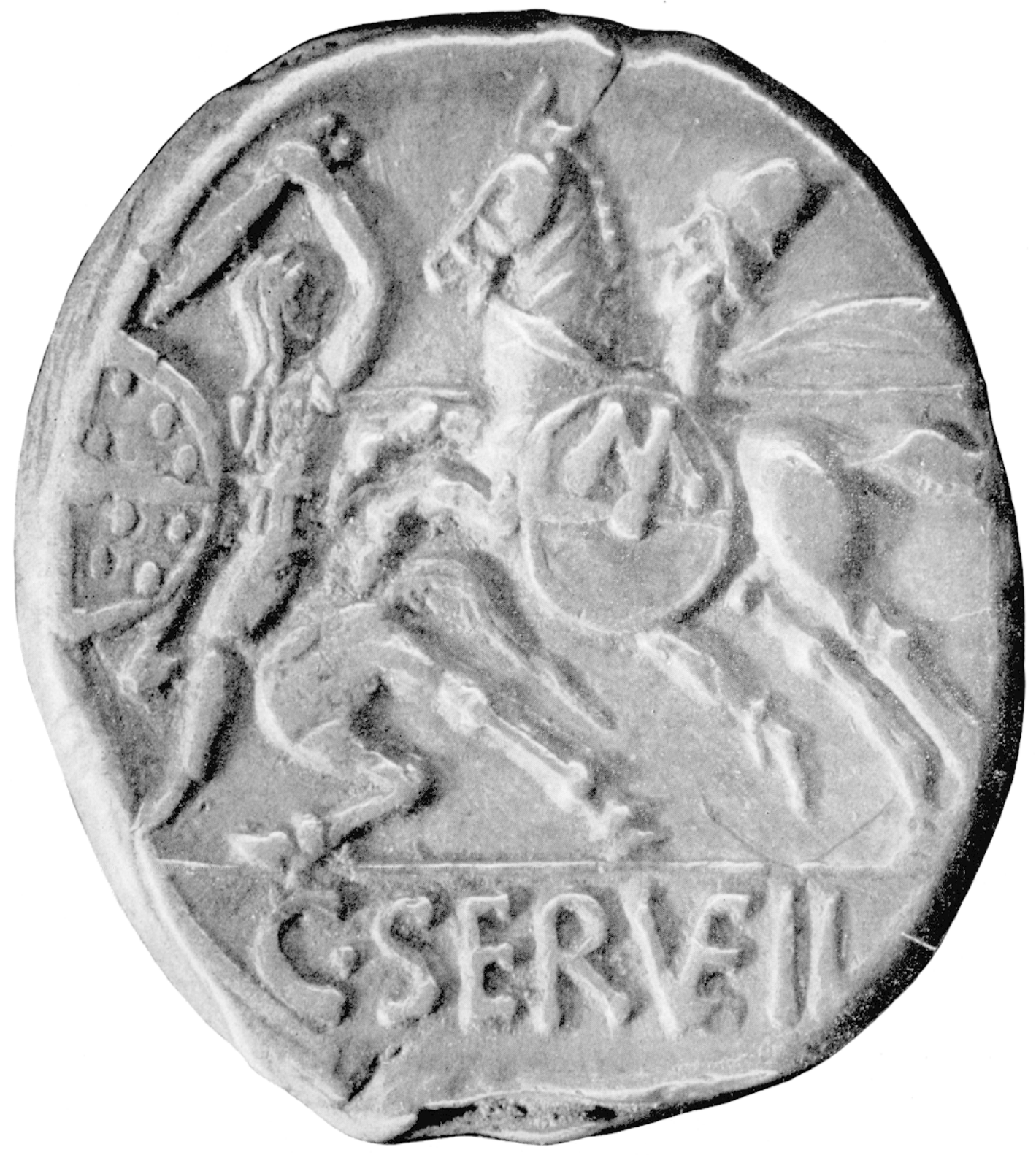

13. -. Monnaie de C. Servilius.

arrache de sa monture, en l'empoignant par les cheveux, un personnage à cheval. Le thème apparaît fréquemment dans les amazonomachies, comme lérimée l'avait déjà reconnu ; comme les Amazones, le personnage désarçonné est vètu de l'exomis et porte au bras gauche une pelte, mais sa poitrine, découverte à droite, ne semble pas féminine. D'autre part, avant même d'être introduit dans le cycle amazonomachique, le motif du guerrier tirant un ennemi de cheval par les cheveux, inventé peut être par Brygos ${ }^{44}$, avait servi à représenter 
Achille capturant Troilos. On le retrouve avec cette signification sur des urnes étrusques ${ }^{45}$, et sur des sarcophages romains du $\mathrm{II}^{\mathrm{e}} \mathrm{s} .{ }^{46}$ Certes le Priamide est habituellement nu, ou couvert d'une simple chlamyde; mais sur le sarcophage de Mantoue, il est pourvu d'un bouclier. I)'autre part, une urne de Volterra complète le groupe, à droite, par l'image d'un Troyen armé qui s'eflorce de défendre le fils de son roi ; Glanicus a recopié le guerrier dans une attitude légèrement différente; le sculpteur volterran a en outre représenté, à gauche d'Achille, une femme ailée pareille à une Niké : nous retrouvons précisément à la même place une victoire tropaeophore sur le mausolée. Il parait donc certain que Glanicus s'est inspiré d'un modèle étrusque, d'où viennent encore les morts et blessés qui apparaissent entre les jambes du cheval. Le fleuve, assis à l'extrémité gauche du relief, est encore pris sûrement à ce modèle : il représentait le Scamandre où Troilos était allé abreuver les chevaux.

Fidèle à sa méthode, Glanicus a enrichi sa composition par divers emprunts : F. Chamoux a reconnu un Lapithe du Parthénon dans le guerrier nu se couvrant d'un bouclier, placé entre le fleuve et la victoire ${ }^{47}$. Le groupe du second plan comprend de gauche à droite : d'abord deux guerriers qui ne prennent aucune part à l'action puis un groupe de trois personnages : un soldat casqué à gauche qu'attaquent, venus de la droite, un personnage vêtu d'un manteau et armé d'un bouclier, et un combattant au casque sans cimier qui charge à la lance, dans l'attitude classique de l'hoplite telle qu'on la trouve déjà au trésor de Siphnos. Quant au personnage qui fuit au premier plan à droite, c'est un dédoublement du "Troyen " de l'urne volterrane.

L'hypothèse d'une influence étrusque sur ce relief devient une certitude si nous considérons le groupe de quatre personnages isolés, à gauche au-dessus de la figure du fleuve. line femme et deux hommes sont réunis autour d'une femme ailée de petite taille qui leur donne lecture d'un volumen. Le génie a l'apparence d'une Niké ; mais la Niké n'a jamais pour attribut le livre ${ }^{4}$. Au contraire, les Étrusques ont représenté avec prédilection une femme ailée, Lasa, Furie ou Parque, tenant et lisant le livre du Destin. Sur un miroir du British Museum ${ }^{49}$ cette entité dressée entre Ajax et Amphiaraos déploie le volumen où l'on lit les noms des deux héros, et le sien propre : Lasa. La Lasa au livre reparaît sur de nombreuses urnes. D'autre part, une urne de Volterra, dont la face principale montre l'apothéose d'un héros, représente sur l'un de ses petits côtés le départ du guerrier, sur l'autre une séance de lecture ou de déclamation à laquelle la Lasa ne participe pas cette fois, mais qui met en scène deux hommes et une femme $\mathrm{e}^{50}$. Le modèle dont s'est servi Glanicus était sans doute une urne dont la face principale représentait la mort de Troilos, et l'un des petits côtés la scène de lecture; il les a réunies en une même composition. L'homme qui se trouve au centre du "groupe de famille" porte sur une tunique un grand manteau agrafé sur

(15) Brunn, 1, 49, 4-51, 8. (Nolterra).

(46) Sarcophage de Mantoue, K. Robert, II, 26, 63. S. Reinach, R. R. III, 52, 2.

(47) Phoibos, VI-VII, 1951-1953, p. 108.

(48) C. A. Piganiol, Recherches sur les jeux romains, p. 118 et n. 3 (confusion de la Lasa et de la Niké, origine itrusque de la Victoire écrivant).

(49) S. de Marinis, Enc. dell' Arte Anlica, IV, p. 488-489, S.v. Lasa, fig. 572. Pour la Lasa au volumen, cf. aussi Brunn, I, 84, 2.

(50) Brunn, II, 2, 65 : S. Reinach, R. R., III, 448, 3. 
l'épaule droite et rejeté sur l'épaule gauche; il semble barbu et a en tous cas les cheveux assez longs et bouclés dans le cou ; la figure est large et les yeux profondément enfoncés. Sa compagne dont le visage est détruit, ètait coiffée d'un chignon roulé et vêtue d'une tunique plisséc aux manches courtes el larges, sur laquelle une stola est drapée en baudrier de l'épaule gauche à la jambe droite. Le troisième personnage, au visage rond et aux cheveux courts s'appuic sur une longue canne ${ }^{51}$.

F. Chamoux a reconnu dans le relief S.-W. un amalgame de la chasse de Cialydon (à droite) et du massacre des Niobides ${ }^{52}$. Or Glanicus n'est pas l'auteur de cette contamination apparemment inexplicable; on la retrouve en effet sur un sarcophage romain du $\operatorname{III}^{\mathrm{e}} \mathrm{s}$. ap. J.-C.. ${ }^{53}$ dont l'auteur ne pouvait évidemment connaître le mausolée des Julii. Cé ne sont pas d'ailleurs les mêmes figures qui ont été retenues par les deux artistes. Du tableau des Niobides, Glanicus a gardé le cheval cabré entraînant un cavalier blessé, le cheval abattu en avant et le pédagogue essayant de protéger un de ses élèves. Sur le sarcophage du Ciapitole, Amphion et un de ses fils à cheval, protégé par un scrvitcur armé, ont été intégrés à gauche, à la chasse calydonienne. De cette dernière Glanicus a éliminé Atalante. Existait-il donc un monument antérieur au mausolée de Saint-Rémy unissant les deux mythes en une même composition, que les deux artistes auraient pris pour modèle? Les deux mythes content la mort pitovable de jeunes chasseurs; il n'en faut pas plus pour qu'ils aient été rapprochés sur des tombeaux d'adolescents ${ }^{54}$. D'autre part, une recherche comparative sur diverses mosaïques de chasses romaines me conduit à partager l'opinion de F. Chamoux sur le prototype de la chasse de Calydon ${ }^{55}$ : il s'agit très vraisemblablement d'une peinture exécutée vers la fin du ive s. av. J.-li. par un artiste de l'école de Pausias et de Philoxénos.

Nous parvenons ainsi à reconstituer de manière assez précise la documentation utilisée par Glanicus : elle se composait : 1) pour la face N.-E. d'une peinture de bataille romaine remontant à la fin du ${ }_{I}{ }^{\mathrm{e}}$ s. et inspirée elle-même par un tableau du temps d'Alexandre; 2) pour la face N.-W. d'une autre peinture du cycle iliaque exécutée probablement elle aussi au premier siècle de l'ère hellénistique ; 3) sur la face S.-W. de deux tableaux sensiblement contemporains des précédents, connus peut-être par l'intermédiaire d'une œuvre italienne qui les avait déjà amalgamés ; 4) sur la face S.-E. enfin d'une urne étrusque du $\mathrm{y}^{\mathrm{e}} \mathrm{s}$. Le choix est déjà significatif : on constate qu'il exclut les œuvres orientales de la fin de l'époque hellénistique, celles notamment de l'école de Pergame. Ce ne peut être l'effet du hasard ou de l'ignorance : le sculpteur de la fontaine et celui de l'autel s'étaient inspirés, quant à eux, de la balustrade du temple mysien d'Athéna Polias. Glanicus connaissait sûrement les galatomachies attalides, ne serait-ce que par l'intermédiaire des copies étrusques; il ne s'est pas servi non plus, d'ailleurs, des monuments proprement helléniques qui glorifient la déroute de Brennos à Delphes : exclusion que la popularité de ces œuvres en Italie rend d'autant plus remarquable.

(51) Cet attribut pourrait désigner un messager.

(52) CRAI, 1945, p. 177-183; Phoibos, l. 1., p. 106. Observations sur la survivance des thèmes helléniques dans la sculplure provençale (Actes du Colloque de Dijon sur l'hellénisation de la Gaule, 1958), p. 37-38.

(53) K. Robert, III, 2, 64, 221. S. Reinach, R. R., III, 194, I.

(54) Sur la signification du mythe des Niobides, en dernier lieu K. Schefold, R.A., 1961, II, p. 189-192.

(55) $B A C, 1951-1960$, p. 189-191. 
On n'explique pas un artiste en faisant la liste des sources où il a puisé son inspiration. I a personnalité de Glanicus s'affirme au contraire en face de ses modèles; s'il a connu et étudié l'art étrusque, son style diffère essentiellement de celui qu'il avait pu apprendre à cette école et $\mathrm{R}$. Bianchi-Bandinelli a cu raison de le souligner ${ }^{56}$; les reliefs des urnes tyrrhéniennes comme ceux du mausolée des Jules, utilisent les trois dimensions de l'espace; mais les personnages étrusques sont détachés comme des statuettes de la masse de l'urne, qui n'est plus qu'un support et un cadre évidé pour laisser pénétrer autour d'eux l'atmosphère naturelle, une scène de théâtre accueillant en elle des acteurs; à Saint-Rémy la pierre est devenue elle-même un espace fictif, comme la toile d'un tableau sous l'effet des couleurs, et les personnages appartiennent tout entiers à cet univers irréel. Cie progrès de l'illusionnisme est d'autant plus notable qu'il se produit au moment même où les écoles romaines réagissent précisément, sous l'influence du néo-atticisme, contre l'attrait de l'expression spatiale : l'œuvre métropolitaine la plus comparable par son sujet à la décoration du mausolée de Glanum, la frise du tombeau de Ciartilius Publicola à Ostie ${ }^{57}$, n'apparaît que comme une interprétation populaire d'un relief classique, et il en est de même de la frise du tombeau d'Eurysacès, malgré le réalisme plébéien du sujet.

Cartilius et Eurysacès, ont voulu illustrer l'activité essentielle de leur existence. Les Julii de Glanum eurent-ils la même intention? On discute du problème depuis plus d'un siècle, et sa solution n'a guère avancé. Une remarque préliminaire est à peine nécessaire : que nous ayons pu déceler, derrière chacun des reliefs du mausolée, un modèle souvent mythologiqur", ne démontre nullement que ces tableaux n'évoquent point des événements contemporains. L'artiste romain transpose constamment des poncifs en représentations historiques; il lui suffit pour cela souvent de revêtir les mannequins qu'il emprunte à son modèle du vêtement porté par les acteurs de l'épisode qu'il veut représenter. Or, plusieurs des personnages de Saint-Rémy ont certainement ainsi changé de costume : sur la face n.-o. par exemple, plusieurs guerriers sont vêtus et armés tout autrement que les Achéens et les Troyens de l'oenochoé de Bernay; nous verrons qu'au n.-e. les cavaliers combattants du groupe central ne portent pas la même armure que Servilius et son adversaire sur la monnaie. Au s.e. au contraire, l' Achille " et le "Lapithe" sont restés nus, et le "Trölos" porte une exomis qui peut bien être son costume d'origine; mais les guerriers du second plan ont un armement tout-à-fait analogue à celui des combattants sur les autres faces. I)u côté s.-o. enfin tous les chasseurs (Niobides ou Cialydoniens) sont nus ou vêtus du costume traditionnel du prototype. Cne première conclusion s'impose donc: Glanicus a " rhabillé " certains de ses personnages seulement ; se donnant ce mal, il est bien peu vraisemblable qu'il soit allé emprunter les costumes qu'il empruntait à un autre modèle. La thèse de $\mathrm{F}$. Chamoux ${ }^{58}$, selon laquelle ces costumes seraient grecs ou hellénistiques, apparaît done peu vraisemblable. Les arguments de détail qui la soutiennent ne nous convainquent d'ailleurs pas : Chamoux considère le casque cornu, porté par un cavalier de la face n.-e., comme la coiffure royale macédonienne ; mais celle-ci apparait sur les deniers de L. Philippus

(56) Sloricità dell'Arle Classica, p. 90.

57) .I. Squarciapino, Scavi di Oslia, III, 1 (Necropoli), p. 169 sqq.

(58) Survivances, p. 36-37. 
comme un "bassinet" cornu, tandis que le casque du cavalier de Saint-Rémy lui emboîte la tête ; rien ne désigne d'ailleurs le personnage comme un roi ; et de toute manière les deux fantassins à casque cornu de la face n.-o. n'ont évidemment rien de princier. Nous verrons tout à l'heure que les boucliers ovales sont bien des scula ct non des clipei circulaires helléniques, vus en perspective, comme l'avaient cru P. Couissin et F. Chamoux. D'ailleurs le bouclier rond apparaît sur le tombeau de Publicola.

A l'analyse très détaillée des armes du Mausolée faite autrefois par Couissin ${ }^{59}$, il n'y aurait, ces réserves faites, guère à ajouter, si nous ne disposions justement des documents nouveaux de l'autel et de la fontaine. Les particularités si originales des cuirasses de la fontaine ne se retrouvent pas au mausolée, bien que les guerriers portent là aussi l'armure " en baril "; même le gorgerin, si fréquent sur les monuments provençaux, n'y apparaît pas. Au contraire la présence dans la frise de la fontaine de trois scula ovales prouve que Couissin s'était trompé en prenant les boucliers ovales du Mausolée pour des clipei déformés par la perspective. Le casque de cette même frise, conforme au modèle réglementairc de l'armée romaine républicaine, est très fréquent sur le tombeau (du moins si l'on ne tient pas compte d'un détail auquel couissin a attaché une importance à notre avis excessive, l'avance, dont tous les casques du mausolée ne sont d'ailleurs pas pourvus) ${ }^{60}$. Parmi les armes de l'autel, ce sont surtout les deux clipei bombés qui sont utiles pour la comparaison avec le monument des Julii : deux des guerriers du relief n.-o. au moins (9 et 11 de la numérotation de ('ouissin) ont en effet le même bouclier, décoré lui aussi d'un « soleil » aux rayons triangulaires ${ }^{61}$.

La comparaison entre la monnaie de Servilius et le combat de cavalerie de la face n.-e. est fort instructive quant à l'ornement ${ }^{62}$. Sur la pièce M. Servilius porte un clipeus rond marqué de l'initiale de son prénom ${ }^{63}$, le casque romain réglementaire à timbre hémisphérique et crinière, et est armé d'une longue lance; son adversaire a, selon les pièces, la téte nue et les cheveux longs ou un casque rond à couvre-nuque très accentué, une cuirasse "baril " très courte, et un énorme bouclier ovale plat, qui parait bien gaulois ; il frappe avec une épée spatulée, à pommeau bouleté. Le sculpteur de Saint-Rémy a échangé les casques des deux champions et supprimé complètement le grand bouclier du cavalier de gauche. Il a donc fait disparaitre une arme (le grand bouclier ovale, qui était employée de son temps en Gaule, et a conservé une épée qui n'est attestée dans cette région et à cette époque par aucune trouvaille archéologique, mais qui arme les guerriers des urnes étrusques. En revanche, il a doté les montures d'une selle, qui se voit parfaitement sur le cheval abattu à gauche : ce harnais inconnu des Grees est au

(59) $R A, 1923,1$, p. 303-321.

(60) l. 1., p. 313.

(61) Ce bouclier apparait fréquemment sur les urnes étrusques : par ex. Brunn Korte, II, 22, $4: 5$. Reinar:h, R. R. III, 542,1 .

(62) Il existe des variantes notables quant à l'armement entre les différentes frappes de cette monnaie ; l'exemplaire du Cabinet des médailles, que je reproduis, diffère de celui du Museo Nazionale Romano, figuré par G. Becatti sur les points suivants : le casque de Servilius est beaucoup plus grand et sans aigrette, la lance a disparu; le guerrier celtique est tête nue et porte les cheveux longs.

(63) L'usage de marquer un bouclier du nom du propriétaire ne parait pas autrement altesté (cf. P. M. Duval, dans l'Arc d'Orange, p. 92). 
contraire utilisé au $\mathrm{I}^{\mathrm{er}}$ s. par les Gaulois et les Romains servant en Gaule ${ }^{64}$. (ilanicus a donc doté la plupart de ses guerriers d'un armement qui était en usage dans l'armée romaine de son temps. Des armes jugées non romaines par Ciouissin, l'épée en particulier, se retrouvent sur les urnes étrusques : elles servaient peut-être encore aux soldats italiques du $\mathrm{I}^{\mathrm{er}}$ s. Ces constatations portent à croire que notre sculpteur entendait représenter des événements contemporains. Malheureusement il est extrêmement difficile d'identifier les adversaires des Romains avec un peuple connu.

Sur la face n.-e. seulement on peut distinguer, outre trois ravaliers romains, porteurs de l'uniforme normal, deux de leurs adversaires qui sont probablement des Gaulois : celui qui se trouve à l'extrême gauche coiffe en effet un casque à cornes caprines, pareil à celui du Gaulois de Berlin ${ }^{65}$. Dans le groupe central, le cavalier de droite a conservé l'attitude (inversée), le casque et la cuirasse de l'adversaire de Servilius Geminus sur la monnaie. Du côté n.-o. s'afirontent deux camps qui sur l'original représentaient respectivement les Troyens et les Achéens; les premiers viennent de la gauche, les seconds de la droite; mais nous trouvons des soldats équipés à la romaine parmi les "Troyens" (le $\mathrm{n}^{0} 2$ de (Couissin) aussi bien que dans les rangs "Achéens" ( $n^{0}$ 5, 6 et 8); des deux guerriers à casque à cornes, l'un (no 4) est, un "Achéen ", l'autre (no 7) probablement un "Troyen ". Au s.-e. Glanicus semble avoir voulu représenter une colonne - dont fait partie "Troïlos" -assaillie des deux côtés par des adversaires placés en embuscade, au passage d'un défilé ; les compagnons de "Troïlos" sont équipés à la romaine. ses adversaires armés et vêtus de manière très hétéroclite : "Achille " parait nu, comme le "Lapithe " tombé près de lui, un des combattants du groupe droit n'a pas de cuirasse. l'autre est équipé à la romaine mais avec un casque sans cimier. De telles inconséquences ne sont pas, en elle-mêmes, tellement surprenantes. Le sculpteur du tombeau de Publicola à Ostie n'est guère plus "réaliste " que son confrère provençal, bien qu'il ne mêle pas comme lui les faits réels au mythe. Il présente croyons-nous, une scène d'enròlement et un débarquement; les conscrits, qui regoivent, au garde à vous, un sculum plus petit que celui du soit-disant "autel d'Ahenobarbus" sont en tunique el nu-tête; dans la galère, un personnage nu, la tête coiffée d'un curieux casque cônique, et protégé par un sculum "en tuile " brandit un javelot, devant un autre combattant en lunique, qui se courre d'une petite parme ronde, analogue à celle dont se servaient les Ibères ${ }^{66}$. Si ces reliefs étaient isolés de leur contexte, personne assurément n'y reconnaîtrait des soldats romains.

Les sculptures de Glanum, - celles du mausolée comme celles des monuments triomphaux - sont donc de piètres documents historiques. Peut-on espérer les éclairer par le recours d'autres témoignages? Cielui des textes étant exclu, le seul recours possible est dans l'épigraphie.

H. Rolland a découvert dans le défilé du sanctuaire deux ex-rotos d'anciens soldats ; l'un d'eux est consacré au dieu Glan, aux Matres Glanicae, et à Fortuna Redux par le

(64) Cf. J. J. Hatt et G. Ch. Picard, L'Arc d'Orange, p. 87.

(65) Déchelette, Manuel d'Archéologie, IV, fig. 734.

(66) Peut-être a-t-on voulu représenter un débarquement (de pirates ?) sur la cóte italienne, repoussé par les milices de Publicola ? Mais il serait étrange qu'on n'ait pas montré le combat et la fuite des envahisseurs. 


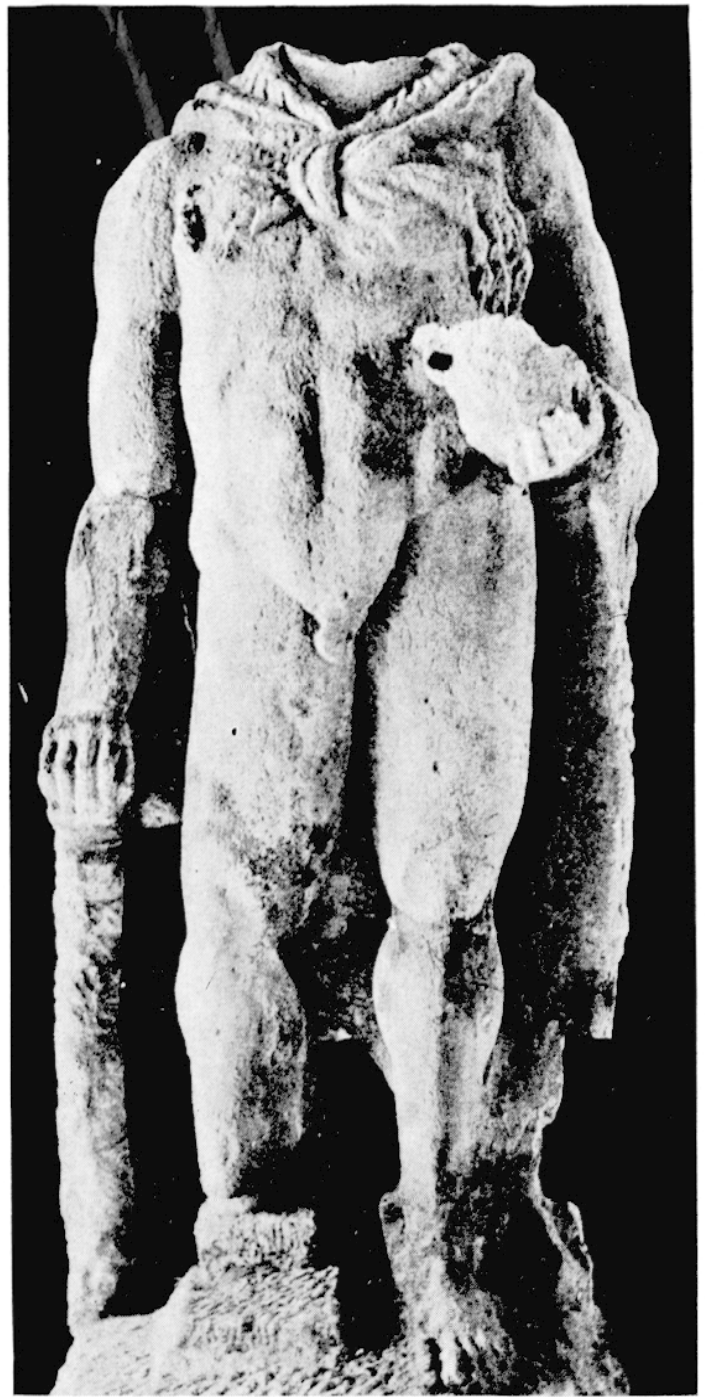

14. -- Glanum. Statue d'Hercule Victor dédiée par loptio Cin. Pompeius Cornulus.

légionnaire M. Licinius Verecundus qui servit dans la légion XXI Rapax entre 9 et 90 ap. J.-C. ${ }^{67}$. L'autre texte pose des problèmes extrêmement complexes ; il s'agit de la dédicace de la statue de culte d'Hercules Victor, vouée par l'option Cn. Pomp(eius) Cornutus, pro salute et reditu G. Licin(i) Macri Irib(uni) et centurionum et militum Glanicorum qui sub vexsillo fuerunt. Le tribun G. Licinius Macer commanda donc une vexillatio, ayant l'effectif d'une cohorte et composée uniquement de Glaniques. Ln tel corps n'a pas sa place dans l'arméc impériale ${ }^{69}$. A partir du règne d'Auguste, les citoyens de Glanum, comme les autres habitants de la Narbonnaise, étaient normalement incorporés dans les légions. D'autre part, il a fallu des circonstances exceptionnelles pour qu'on mobilise d'un coup plusieurs centaines d'hommes dans une aussi petite ville. Une telle levée a pu être décidée par Ciésar, en - 49, ou par l'un des maitres de la Gaule entre -44 et - 31. On peut supposer aussi à la rigueur qu'elle ait été décrétée plus tard pendant une période de guerres civiles, en $+68+69$ par exemple; cette seconde hypothèse est moins probable, car les termes dont se sert Cornutus impliquent que la vexillation a servi assez longtemps pour qu'il ait pu rentrer au pays pendant que ses compagnons d'armes demeuraient sous les drapeaux. Mais l'inscription de Cornutus peut-elle remonter au $\mathrm{r}^{\mathrm{er}} \mathrm{s}$. ? Écrite dans une capitale extrêmement régulière, elle comporte à côté d'une orthographe légèrement archaïsante - vexsillo - des ligatures

(67) Glanum Il, p. 88 et pl. 30,3 .

(68) L.a XXI Rapax, constituée en +9, après le désastre de Varrus, se mutina en 97 et fut dissoute (J. J. Hatt, Histoire de la Gaule Romaine, p. 168-169).

(69) L'expression qui sub vexil [lo praeleribunt?] se rencontre dans une inscription malheureusement très mutilée découverte par Ch. Saumagne à Sidi Amara en Tunisie (Dubiez et Saumagne, BAC, 1928-1929, p. 226-233 = A. Merlin, Inscr. Lat. de Tunisie, 625) qui parait reproduire le texte d'un édit impérial du $~{ }^{e} \mathrm{~s}$. relatif au ravitaillement des troupes en marche. Sur l'expression, cf. le commentaire de Ch. Saumagne, p. 230, n. 2 qui renvoie notamment à R. Cagnat, Armée Rom. d'Afrique, p. 14 et à Max Meyer, Vexillum, Strasbourg, 1910, que je n'ai pu encore consulter, n'ayant découvert cette référence que pendant la correction des épreuves du présent article. Én attendant une recherche plus poussée, je me borne donc à indiquer que la situation de la vexillalio glanica de Licinius .Macer paraît différente de celle des corps visés par l'édit de Sidi Amara. Ceux-ci pourraient être soit des vexillationes tironum, soit des vexillations légionnaires, soit, comme l'indique $\mathrm{Ch}$. Saunagne, des vexillations de spécialistes, tandis que l'unité glanique paralt bien être une milice locale. 
et surtout une abréviation de gentilice --. Pomp(eius) - qui sont insolites avant le $\mathrm{II}^{\mathrm{e}} \mathrm{s}$, liependant on retrouve des ligatures et des abréviations, celles-là tout-à-fait anormales. sur les autres autels dédiés à Hercule dans le sanctuaire, qui ne paraissent pas postérieurs au $\mathrm{i}^{\mathrm{er}} \mathrm{s}$. Surtout la dédicace n'est certainement pas plus récente que la statue sur la base de laquelle elle était gravée (fig. 14). Or cette image d'Hercule, massive, à la lourde musculature schématiséc géométriquement, à la main à peine ébauchée, est une cuvre d'art populaire, encore proche du dieu accroupi provenant du sanctuaire celtoligure. Le sculpteur de la fontaine, qui aime lui aussi les fortes saillies musculaires, génératrices de jeux d'ombre et de lumière, connaissait beaucoup mieux l'anatomie que l'auteur de l'Hercule, qui est, selon toute vraisemblance, son devancier. Il est donc permis de dater l'ex-voto de Cin. Pompeius Cornutus entre - 50 et - 40 environ, juste avant le temple de Valetudo, qui fait pendant au fanum d'Hercule, de l'autre côté de la source sacrée. Nous pouvons admettre qu'une mobilisation massive de la population glanique fut ordonnée par César lorsqu'il appela à lui toutes les forces de la Gaule, en — 49. Le chef du détachement, un Licinius, avait obtenu le droit de cité par l'entremise d'un des Crassi ; un des sousofficiers tenait le sien de Pompée; d'autres notables engagés furent naturalisés par César lui-même. L'un de ces derniers, revenu mourir chez lui, au moment où un nouveau César rendait la paix à l'empire, fut honoré par ses fils du somptueux mausolée qui devait longtemps conserver, seul avec l'arc élevé quelques années plus tard à côté, le souvenir de la petite cité. Ie sculpteur, qui avait appris son métier en Italie, à l'école des derniers ateliers étrusques, illustra le socle du monument de scènes mi-allégoriques, mi-historiques qui exaltaient toutes la virlus du défunt : une chasse au sanglier, dans laquelle, comme plus tard un des Laberii d'Lthina en Afrique sur l'une des mosaïques de son domaine, Julius assumait l'apparence de Véléagre; trois scènes de bataille, dont deux reprenaient des thèmes de l'Iliade, tandis que la troisième transposait un tableau célèbre de l'art triomphal romain. Les épisodes avaient-ils été choisis parce qu'ils présentaient quelque analogie avec les exploits réels de Julius, ou simplement pour suggérer que sa vaillance ne le cédait ni à celle d'Achille, ni à celle de Méléagre, ni à celle de Servilius ? Cette dernière interprétation nous semble la plus probable, mais le problème ne pourra être résolu que lorsque nous serons mieux renseignés - si du moins nous pouvons espérer l'être un jour - sur la signification des scènes de bataille mythologiques et historiques dans l'art funéraire étrusque. Il paraît certain en tout cas que le mausolée de Saint-Rémy doit être interprété en fonction d'une mystique eschatologique. Cette exégèse s'impose en effet si nous considérons le lien logique qui unit les trois parties du monument, et leur assigne une signification précise. Chacun de ces trois éléments constitue architecturalement un monument complet : le socle est un autel, comme l'indique la guirlande sculptée au-dessus des scènes de bataille ; l'étage moyen, un arc de triomphe; la "lanterne " supérieure, une tholos, un temple par conséquent. D'autre part, ces monuments correspondent tous trois à l'une des étapes de la destinée de Julius : le socle évoque sa vie terrestre hérö̈que, l'arc avec ses portes sommées du gorgoneion et sa frise marine symbolise l'entrée triomphale dans l'au-delà, l'arrivée dans les îles célestes des Bienheureux. La thloos enfin, qui abrite les statues, est évidemment l'image du paradis ouranien. Le principe d'une telle progression est lié au type du mausolée 
tour, probablement dès ses origines anatoliennes : nous en retrouvons en effet l'expression sur le mausolée punique de Dougga, antérieur de plus d'un siècle à celui de Saint-Rémy, et appartenant à un tout autre domaine culturel : les quadriges et les Sirènes du troisième étant les moyens du voyage du mort, depuis la chambre funéraire jusqu'au séjour astral que symbolise le pyramidion surmonté de la statue léonine ${ }^{70}$.

Nais cette interprétation eschatologique du mausolée-tour, si elle s'est maintenue aisément dans les régions, comme l'Afrique punique, que dominait une religion transcendantale, ne devait trouver d'écho dans la Rome de la fin de la République, qu'auprès des cercles isiaques ${ }^{71}$, pythagoriciens, ou bacchants par exemple, qui admettaient les idées d'immortalité et de salut. Les études qui ont été faites, à Ostie, sur la genèse des tombeaux monumentaux ${ }^{72}$, l'analyse du décor des mausolées d'époque césarienne ou augustéenne à Rome et dans ses environs, n'autorisent guère à voir dans ces sépultures autre chose que des mémoriaux destinés à entretenir le souvenir du défunt parmi les vivants. La comparaison du mausolée de Cartilius Publicola et de celui des Julii est une fois de plus instructive à cet égard. Le souci du magistrat ostien est de magnifier sa carrière municipale, par l'inscription et les faisceaux qui l'encadrent; ses exploits militaires qui forment le sujet de la frise n'intervenant que pour compléter le compte rendu de cette existence exemplaire. Le notable glanique n'a jugé utile de rappeler aucune des dignités qu'il avait pu recevoir dans sa patrie ou à l'armée : ses exploits apparaissent seulement comme une préparation à la vie future, et c'est pourquoi ils sont présentés dans une atmosphère mythique, qui noie presque entièrement l'élément historique. Ciette atmosphère mythique et mystique est près de celle que créait l'art funéraire étrusque. Elle renaîtra à Rome, à la fin du ier s. ap. J.-C., avec les grands sarcophages à sculptures symboliques. Le mausolée de Saint-Rémy établit la liaison entre ces deux familles monumentales, de telle manière qu'il apparaît tantôt en retard et tantôt en avance sur son temps : reprenant d'une part, indiscutablement, le langage et les procédés des imagiers tyrrhéniens ; exprimant d'autre part une théologie de la Virtus semblable à celle que nous lisons sur la colonne Trajane et sur les sarcophages de bataille de l'époque antonine, mais qui transparaît, dès le $\mathrm{I}^{\mathrm{er}} \mathrm{s}$., au travers du modeste décor des stèles funéraires de l'armée rhénane. Cette position d'intermédiaire est d'ailleurs, comme nous l'avons montré à propos de l'arc d'Orange, celle de l'art romano-provençal tout entier, témoin de la résistance opposée par l'esprit italique au classicisme rationaliste augustéen.

G. Ch. Picard.

(70) C.f. Religions de l'Afrique Antique, p. 9091.

(71) Sur l'Isiasme en Italie au rer s., voir en dernier lieu V. Tran tam Tinh, Le culle d' Isis à Pompéi

(72) M. Squarciapino, l. $l$. 